\title{
Neural Variability Limits Adolescent Skill Learning
}

\author{
(D) Melissa L. Caras ${ }^{1}$ and Dan H. Sanes ${ }^{1,2,3,4}$ \\ ${ }^{1}$ Center for Neural Science, Departments of ${ }^{2}$ Psychology, ${ }^{3}$ Biology, New York University, New York, New York, 10003, and ${ }^{4}$ Neuroscience Institute, NYU \\ Langone Medical Center, New York, New York, 10016
}

Skill learning is fundamental to the acquisition of many complex behaviors that emerge during development. For example, years of practice give rise to perceptual improvements that contribute to mature speech and language skills. While fully honed learning skills might be thought to offer an advantage during the juvenile period, the ability to learn actually continues to develop through childhood and adolescence, suggesting that the neural mechanisms that support skill learning are slow to mature. To address this issue, we asked whether the rate and magnitude of perceptual learning varies as a function of age as male and female gerbils trained on an auditory task. Adolescents displayed a slower rate of perceptual learning compared with their young and mature counterparts. We recorded auditory cortical neuron activity from a subset of adolescent and adult gerbils as they underwent perceptual training. While training enhanced the sensitivity of most adult units, the sensitivity of many adolescent units remained unchanged, or even declined across training days. Therefore, the average rate of cortical improvement was significantly slower in adolescents compared with adults. Both smaller differences between sound-evoked response magnitudes and greater trial-to-trial response fluctuations contributed to the poorer sensitivity of individual adolescent neurons. Together, these findings suggest that elevated sensory neural variability limits adolescent skill learning.

Key words: adolescence; auditory cortex; development; internal noise; perceptual learning; practice

\section{Significance Statement}

The ability to learn new skills emerges gradually as children age. This prolonged development, often lasting well into adolescence, suggests that children, teens, and adults may rely on distinct neural strategies to improve their sensory and motor capabilities. Here, we found that practice-based improvement on a sound detection task is slower in adolescent gerbils than in younger or older animals. Neural recordings made during training revealed that practice enhanced the sound sensitivity of adult cortical neurons, but had a weaker effect in adolescents. This latter finding was partially explained by the fact that adolescent neural responses were more variable than in adults. Our results suggest that one mechanistic basis of adult-like skill learning is a reduction in neural response variability.

\section{Introduction}

The refinement of sensory and motor abilities with practice- $-\mathrm{a}$ process known as skill learning - is critical for the maturation of many complex behaviors, from walking to language acquisition (Kuhl, 2000; Kuhl et al., 2006; Adolph et al., 2018). One specific form of skill learning-perceptual learning-is characterized by an improvement in the ability to identify, detect, or discriminate stimuli and plays an important role in shaping sensory perception (for review, see Gibson, 1953; Wright and Zhang, 2009; Lu et

\footnotetext{
Received Nov. 8, 2018; revised Jan. 24, 2019; accepted Jan. 26, 2019.

Author contributions: M.L.C. and D.H.S. designed research; M.L.C. performed research; M.L.C. analyzed data; M.L.C. and D.H.S. wrote the paper.

This work was supported by National Institutes of Health Grants R01-DC-014656 (D.H.S.) and F32-DC-014376 (M.L.C). We thank Derek Wang and Stephen Young for assistance with data collection and histology. We also thank Dr. Daniel Stolzberg, Dr. Jonathan Fritz, and members of the Sanes laboratory for constructive comments.

The authors declare no competing financial interests.

Correspondence should be addressed to Melissa L. Caras at caras@nyu.edu.

https://doi.org/10.1523/JNEUROSCI.2878-18.2019

Copyright $\odot 2019$ the authors
}

al., 2011; Irvine, 2018). While neurophysiological experiments firmly establish the importance of cortical plasticity in adult perceptual learning (Recanzone et al., 1992, 1993; Crist et al., 2001; Schoups et al., 2001; Beitel et al., 2003; Bao et al., 2004; Yang and Maunsell, 2004; Polley et al., 2006; Raiguel et al., 2006; Li et al., 2008; Adab and Vogels, 2011; Gu et al., 2011; Powers et al., 2012; Yan et al., 2014; Caras and Sanes, 2017), the neural mechanisms that support this process during development are uncertain. Here we report that the neural correlates of perceptual learning differ between juvenile and adult animals as they train and improve on an auditory task.

A growing number of behavioral studies reveal that skilllearning capabilities continue to mature throughout childhood and adolescence (Huyck and Wright, 2011, 2013; van der Schaaf et al., 2011; Pattwell et al., 2012; Chihak et al., 2014; Decker et al., 2015; Lukács and Kemény, 2015; Knoll et al., 2016). For example, Huyck and Wright (2011) trained adolescent (11-14 years old) and adult humans on a temporal interval discrimination task. While adults uniformly improved with practice, reducing their 
discrimination thresholds by more than half after $10 \mathrm{~d}$ of training, adolescent performance remained steady, or even degraded, as training progressed. Indeed, the development of skill learning often follows a nonlinear trajectory, displaying a transient stabilization or reduction of capabilities during adolescence (van der Schaaf et al., 2011; Pattwell et al., 2012; Lukács and Kemény, 2015). Together, these behavioral findings suggest that children, adolescents, and adults may each engage distinct neural strategies to enhance their perceptual skills.

This study asks whether auditory cortical processing does, indeed, differ between juvenile and adult gerbils as they train and improve on the same auditory task. We found that perceptual learning follows a nonlinear developmental trajectory, such that adolescents learned more slowly than either young or adult animals. We made wireless extracellular recordings from the auditory cortex of a subset of adolescent and adult animals as they underwent perceptual training. Consistent with our behavioral observations, we found that neural sensitivity improved more slowly in adolescent animals. This delay could be explained by an elevated trial-to-trial response variability and a weaker effect of training on sound-evoked response magnitudes in adolescents. Collectively, our results indicate that adolescence is characterized by a period of sensory instability that limits perceptual learning.

\section{Materials and Methods}

\section{Experimental subjects}

A total of 52 Mongolian gerbils (Meriones unguiculatus) raised from commercially obtained breeding pairs (Charles River Laboratories) were used in this study. After weaning at postnatal day 30 (P30), animals were group housed with littermates, and were separated into samesex cages containing two to six animals by P60. All animals were exposed to a $12 \mathrm{~h}$ light/dark cycle, and had free access to food and water unless otherwise noted. All procedures were approved by the Institutional Animal Care and Use Committee at New York University.

Animals began training at three different ages (Fig. 1C), with each group consisting of animals from multiple litters. "Young" animals $(n=19)$ began procedural training at P35 and perceptual training at P38. "Adolescent" animals $(n=17)$ began procedural training between P41 and P49 (mean $=$ P47) and perceptual training between P44 and P52 $($ mean $=$ P50). All "adult" animals $(n=16)$ began procedural training at P96 or later $($ mean $=$ P111 $)$ and perceptual training at P100 or later $($ mean $=$ P116). A subset of the adolescent $(n=6)$ and adult animals $(n=4)$ was also used for extracellular electrophysio-

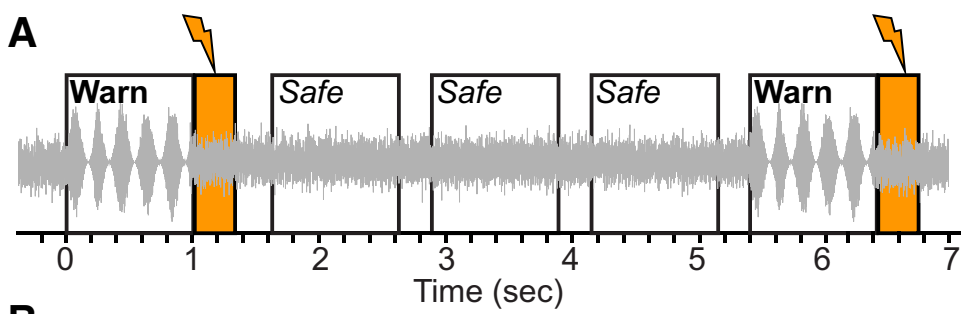

B
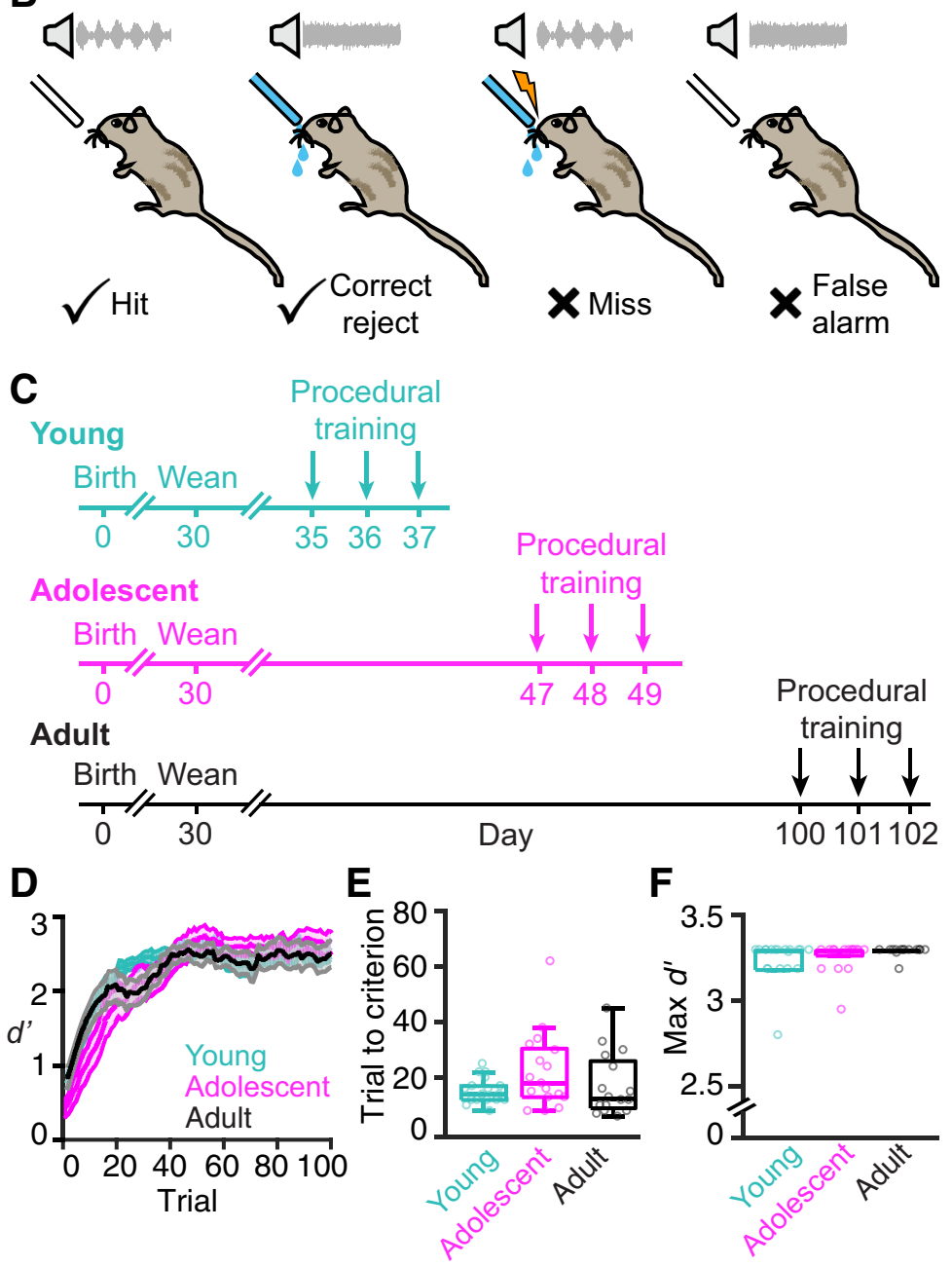

Figure 1. Age does not affect the rate or extent of procedural learning. $\boldsymbol{A}$, Trial structure and timing of aversive conditioning task. Animals were required to detect when a safe stimulus (unmodulated broadband noise) smoothly transitioned to a warn stimulus ( $0 \mathrm{~dB}$ at $100 \%$ depth AM noise; $5 \mathrm{~Hz}$ rate). Warn trials were immediately followed by an aversive shock ( $300 \mathrm{~ms}$, orange bars), which could be avoided by withdrawing from the spout. Warn trials were separated by three to five safe trials, such that the onset of each warn stimulus was unpredictable. $\boldsymbol{B}$, Animals were trained to drink continuously from a spout of water while in the presence of the safe stimulus. Maintaining spout contact during safe trials was therefore scored as a correct reject, whereas withdrawing from the spout was considered an incorrect false alarm. Withdrawing from the spout upon presentation of the AM warn stimulus was scored as a correct hit. Maintaining contact with the spout during the warn stimulus was considered an incorrect miss and punished with a shock. C, Experimental timeline of procedural training. All animals were weaned at P30. Subjects had $3 \mathrm{~d}$ of procedural training before progressing to perceptual training (Fig. 2). Some adults required additional sessions due to poor initial motivation to participate in the task, likely due to a robust (mature) ability to retain water. Ages depicted here are exact for young animals, and approximate for adolescent and adult animals (for details, see Materials and Methods). D, Behavioral performance $\left(d^{\prime}\right)$ was calculated as a function of trial number collapsed across all procedural training sessions using a 15-trial-wide sliding window. Data are depicted as the mean \pm SEM. For this and subsequent panels in this figure, the number of subjects in each age group are as follows: young, $n=19$; adolescent, $n=17$; adult, $n=16$. $\boldsymbol{E}$, The number of trials to reach training criterion $\left(d^{\prime} \geq\right.$ 1.5) is plotted for each animal as a function of age group. Horizontal lines represent median values, boxes represent 25 th and 75 th percentiles, and whiskers represent $1.5 \times$ the interquartile range. $\boldsymbol{F}$, The maximum $\boldsymbol{d}^{\prime}$ value achieved during procedural training is plotted for each animal as a function of age group. Data are depicted as in $\boldsymbol{E}$. Note that boxes and whiskers are present, but may be too small to see. Hit rates and false alarm rates were adjusted to avoid $d^{\prime}$ values that approach Inf, such that the maximum $d^{\prime}$ possible on this task was 3.29 . 
logical recordings that took place throughout perceptual training. Age group classifications were based on previous reports that the onset of the pubertal (i.e., "adolescent") period in the gerbil occurs between P42 and P57, and sexual maturity is not achieved until approximately P90 (Siegford et al., 2003; Pinto-Fochi et al., 2016).

Neurophysiological recordings were not obtained from young animals due to the technical difficulties involved. Specifically, to allow for sufficient recovery time after electrode implantation, yet still to begin perceptual testing by $\sim \mathrm{P} 38$, animals would have needed to undergo surgery at $\sim$ P23 (7 d before we typically wean the animals). At that age, the skull sutures are not fully closed, and the bone is too soft to allow for the hardware implantation required to stabilize the electrode. In addition, the gerbil brain changes its shape dramatically between P23 and P30, such that even if the electrode successfully targets auditory cortex during the initial surgery, the electrode may shift out of auditory cortex by the time recordings commence. We therefore restricted the neurophysiology experiments to adolescent and adult animals.

As expected, animal weights increased significantly with age (oneway ANOVA, $\left.F_{(2,49)}=163.60, p<0.001\right)$. On the first day of procedural training, young animals weighed an average of $27.4 \mathrm{~g}$ (range, 23.3-32.5 g), adolescents weighed an average of $41.6 \mathrm{~g}$ (range, 35.6$48.7 \mathrm{~g}$ ), and adults weighed an average of $63.1 \mathrm{~g}$ (range, 49.7-77.2 g). These weights are consistent with previously published findings (Clark and Galef, 1980).

Some aspects of the data presented in this manuscript were published previously (Caras and Sanes, 2015, 2017). For a full breakdown of the contribution of each subject to the current and previous studies, see Table 1.

\section{Method details}

Behavioral training. To assess the effect of development on perceptual learning and its neural correlates, all animals were trained and tested on a go/no go aversive conditioning paradigm, as described previously (Caras and Sanes, 2015, 2017). Briefly, animals were placed on controlled water access and began procedural training, during which an ongoing "safe" stimulus (i.e., unmodulated broadband noise; $2.5-20 \mathrm{kHz} ; 12 \mathrm{~dB} /$ octave rolloff; $60 \mathrm{~dB}$ SPL) occasionally smoothly transitioned to a "warn" stimulus [sinusoidally amplitude-modulated (AM) noise; $5 \mathrm{~Hz}$ modulation rate; $100 \%$ depth, $1 \mathrm{~s}$ duration]. Warn stimuli were immediately followed by a mild aversive shock (Colbourn Instruments) delivered through the metal lick spout for $300 \mathrm{~ms}$. Because pain sensitivity varies across individual animals (Mogil, 1999), the shock level (0.5-3 mA) was adjusted on a subject-by-subject basis to reliably elicit spout withdrawal without dissuading the animal from promptly recommencing drinking. A minimum of three and a maximum of five safe trials (each of $1 \mathrm{~s}$ duration) occurred between each warn trial (Fig. 1A), such that the onset of each warn stimulus was unpredictable. Sound delivery and data acquisition were controlled by an RZ6 Multifunction Processor (Tucker Davis Technologies) controlled by custom Python scripts (written by Dr. Bradley Buran, Oregon Health \& Science University, Portland, OR).

To quantify behavioral performance, we monitored the contact of the animal with the spout within the final $100 \mathrm{~ms}$ of every trial. Breaking contact for $>50$ ms was considered a spout "withdrawal" and was scored as a correct "hit" on warn trials and as an incorrect "false alarm" on safe trials (Fig. 1B). Conversely, maintaining contact with the spout for $>50$ ms was scored as an incorrect "miss" on warn trials, and a "correct reject" on safe trials. Hit rates (the proportion of warn trials that elicited a hit) and false alarm rates (the proportion of safe trials that elicited a false alarm) were converted into the signal detection metric $d^{\prime}$ (Green and Swets, 1966) as follows:

$$
d^{\prime}=z \text { (hit rate) }-z \text { (false alarm rate). }
$$

The location of the criterion $(C)$ was used as an indicator of response bias (Macmillan and Creelman, 1990) and was calculated as follows:

$$
\left.\left.C=-0.5^{\star}(z \text { (hit rate })+z \text { (false alarm rate }\right)\right) .
$$

To avoid $d^{\prime}$ and $C$ values that approach infinity, we set a floor (0.05) and a ceiling $(0.95)$ on hit rates and false alarm rates.
Table 1. Full breakdown of each subject's contribution to current and previous

\begin{tabular}{|c|c|c|c|}
\hline Animal ID & Age group & Current experiment & Original dataset \\
\hline 241872 & Young & Behavior (Figs. 1, 2) & Current manuscript \\
\hline 241873 & Young & Behavior (Figs. 1, 2) & Current manuscript \\
\hline 241874 & Young & Behavior (Figs. 1, 2) & Current manuscript \\
\hline 241876 & Young & Behavior (Figs. 1, 2) & Current manuscript \\
\hline 241877 & Young & Behavior (Figs. 1, 2) & Current manuscript \\
\hline 246766 & Young & Behavior (Figs. 1, 2) & Current manuscript \\
\hline 246767 & Young & Behavior (Figs. 1, 2) & Current manuscript \\
\hline 246768 & Young & Behavior (Figs. 1, 2) & Current manuscript \\
\hline 246769 & Young & Behavior (Figs. 1, 2) & Current manuscript \\
\hline 246770 & Young & Behavior (Figs. 1, 2) & Current manuscript \\
\hline 246771 & Young & Behavior (Figs. 1, 2) & Current manuscript \\
\hline 246772 & Young & Behavior (Figs. 1, 2) & Current manuscript \\
\hline 191694 & Young & Behavior (Figs. 1, 2) & Caras and Sanes, 2015 (Figs. 3A, 4-6) \\
\hline 191696 & Young & Behavior (Figs. 1, 2) & Caras and Sanes, 2015 (Figs. 3A, 4-6) \\
\hline 191697 & Young & Behavior (Figs. 1, 2) & Caras and Sanes, 2015 (Figs. 3A, 4-6) \\
\hline 192794 & Young & Behavior (Figs. 1, 2) & Caras and Sanes, 2015 (Figs. 3A, 4-6) \\
\hline 192797 & Young & Behavior (Figs. 1, 2) & Caras and Sanes, 2015 (Figs. 3A, 4-6) \\
\hline 199427 & Young & Behavior (Figs. 1, 2) & Caras and Sanes, 2015 (Figs. 3A, 4-6) \\
\hline 199430 & Young & Behavior (Figs. 1, 2) & Caras and Sanes, 2015 (Figs. 3A, 4-6) \\
\hline 203435 & Adolescent & Behavior (Figs. 1, 2) & Caras and Sanes, 2015 (Fig. 3B) \\
\hline 203436 & Adolescent & Behavior (Figs. 1, 2) & Caras and Sanes, 2015 (Fig. 3B) \\
\hline 203437 & Adolescent & Behavior (Figs. 1, 2) & Caras and Sanes, 2015 (Fig. 3B) \\
\hline 203444 & Adolescent & Behavior (Figs. 1, 2) & Caras and Sanes, 2015 (Fig. 3B) \\
\hline 203445 & Adolescent & Behavior (Figs. 1, 2) & Caras and Sanes, 2015 (Fig. 3B) \\
\hline 203446 & Adolescent & Behavior (Figs. 1, 2) & Caras and Sanes, 2015 (Fig. 3B) \\
\hline 203447 & Adolescent & Behavior (Figs. 1, 2) & Caras and Sanes, 2015 (Fig. 3B) \\
\hline 207341 & Adolescent & Behavior (Figs. 1, 2) & Caras and Sanes, 2015 (Fig. 3B) \\
\hline 207342 & Adolescent & Behavior (Figs. 1, 2) & Caras and Sanes, 2015 (Fig. 3B) \\
\hline 207343 & Adolescent & Behavior (Figs. 1, 2) & Caras and Sanes, 2015 (Fig. 3B) \\
\hline 207344 & Adolescent & Behavior (Figs. 1, 2) & Caras and Sanes, 2015 (Fig. 3B) \\
\hline 200349 & Adult & Behavior (Figs. 1, 2) & Caras and Sanes, 2015 (Fig. 30$)$ \\
\hline 200858 & Adult & Behavior (Figs. 1, 2) & Caras and Sanes, 2015 (Fig. 30 \\
\hline 200859 & Adult & Behavior (Figs. 1, 2) & Caras and Sanes, 2015 (Fig. 30$)$ \\
\hline 200860 & Adult & Behavior (Figs. 1, 2) & Caras and Sanes, 2015 (Fig. 30$)$ \\
\hline 200861 & Adult & Behavior (Figs. 1, 2) & Caras and Sanes, 2015 (Fig. 30 \\
\hline 201080 & Adult & Behavior (Figs. 1, 2) & Caras and Sanes, 2015 (Fig. 30$)$ \\
\hline 201081 & Adult & Behavior (Figs. 1, 2) & Caras and Sanes, 2015 (Fig. 30$)$ \\
\hline 209451 & Adult & Behavior (Figs. 1, 2) & Caras and Sanes, 2015 (Fig. 30$)$ \\
\hline 209452 & Adult & Behavior (Figs. 1, 2) & Caras and Sanes, 2015 (Fig. 30$)$ \\
\hline 209455 & Adult & Behavior (Figs. 1, 2) & Caras and Sanes, 2015 (Fig. 30 \\
\hline 209456 & Adult & Behavior (Figs. 1, 2) & Caras and Sanes, 2015 (Fig. 30$)$ \\
\hline 209457 & Adult & Behavior (Figs. 1, 2) & Caras and Sanes, 2015 (Fig. 30 \\
\hline 191699 & Adolescent & Ephys + Behavior (Figs. 1-6) & Current manuscript \\
\hline 192800 & Adolescent & Ephys + Behavior (Figs. 1-6) & Current manuscript \\
\hline 201700 & Adolescent & Ephys + Behavior (Figs. 1-6) & Current manuscript \\
\hline 201701 & Adolescent & Ephys + Behavior (Figs. 1-6) & Current manuscript \\
\hline 203388 & Adolescent & Ephys + Behavior (Figs. 1-6) & Current manuscript \\
\hline 203389 & Adolescent & Ephys + Behavior (Figs. 1-6) & Current manuscript \\
\hline 217821 & Adult & Ephys + Behavior (Figs. 1-6) & Caras and Sanes, 2017 (Figs 1-3) \\
\hline 221955 & Adult & Ephys + Behavior (Figs. 1-6) & Caras and Sanes, 2017 (Figs 1-3) \\
\hline 222724 & Adult & Ephys + Behavior (Figs. 1-6) & Caras and Sanes, 2017 (Figs 1-3) \\
\hline 222725 & Adult & Ephys + Behavior (Figs. 1-6) & Caras and Sanes, 2017 (Figs 1-3) \\
\hline
\end{tabular}
studies

Ephys, Electrophysiology.

Animals were judged to be fully trained on the task after performing at least three procedural training sessions, and achieving a $d^{\prime} \geq 1.5$ in at least one session. While the majority of animals reached this training criterion within three sessions, some adults and one adolescent required additional training sessions, usually due to insufficient motivation to perform (i.e., a lack of thirst). During the final procedural training session, and throughout all subsequent perceptual training sessions, the sound intensity was set at $45 \mathrm{~dB}$ SPL.

After animals were fully trained on the task, they began perceptual training, during which a range of AM depths was presented to each animal to elicit a psychometric function. Because the decision axis for AM depth is logarithmic (Wakefield and Viemeister, 1990), depths are presented throughout this manuscript on a decibel scale (relative to 
$100 \%$ depth). Thus, $0 \mathrm{~dB}$ refers to fully modulated (100\% depth) AM noise, and increasingly negative numbers refer to shallower depths. These decibel (re: 100\% depth) values are not to be confused with decibel SPL values, which indicate the root mean square intensity of the stimulus.

During each perceptual training session, five AM depths, chosen to bracket each individual's detection threshold (defined as the depth at which $d^{\prime}=1$ ), were presented in descending order (interspersed with three to five safe trials, as described above). Values were adjusted as needed during each session to maintain threshold bracketing. All animals received 5-10 perceptual training sessions (except for one animal used for electrophysiological recording, for which we were only able to obtain data over 3 sessions). The starting stimulus values for the second session and beyond were determined by the behavioral performance of the animal during the previous session. Maintaining threshold bracketing within and across sessions allowed for the acquisition of full psychometric functions, but required the presentation of some depths that were unlikely to be detected by the animal. Delivering aversive feedback during such trials would likely cause the animal to peck intermittently at the spout (which would artificially inflate the false alarm rate) or to avoid drinking all together. To avoid these possibilities, the shock was delivered only for the highest three depths presented at any given time. This approach was validated by a previous study (Buran et al., 2014a), which confirmed that the animals do not become additionally conditioned to the presence or absence of the shock.

To quantify psychometric thresholds, hit rates were plotted as a function of modulation depth and fit with a cumulative Gaussian function using a maximum likelihood approach. Fitting was performed using the open-source package psignifit 4 for MATLAB (Schütt et al., 2016) using the default priors of the package. After fits were generated, they were transformed to the signal detection metric $d^{\prime}$. For each fit, threshold was defined as the AM depth at which $d^{\prime}=1$.

Neurophysiology. Each animal used for electrophysiological recordings was implanted with a 16-channel silicone probe array (catalog \#A4x4-4 mm-200-200-1250-H16_21 mm; NeuroNexus) fixed to a custom-made microdrive in left core auditory cortex as previously described (Caras and Sanes, 2017; see Fig. 3B). We targeted left auditory cortex because it is more sensitive to time-varying stimuli than the right auditory cortex (Heffner and Heffner, 1984; Jamison et al., 2006; Wetzel et al., 2008). Adolescents $(n=6)$ were implanted between P30 and P34. Adults $(n=$ 4) were implanted between P86 and P148. Animals were allowed to recover for $3 \mathrm{~d}$ before being checked for neural activity and were allowed a full week of recovery before being placed on controlled water access.

Neural recordings were made from awake, behaving animals during perceptual training sessions. Extracellular neural activity was acquired via a 15-channel wireless headstage and receiver (model W16, Triangle Biosystems). Signals were preamplified, digitized at $24.414 \mathrm{kHz}$ (TB32 digitizer, Tucker Davis Technologies), and fed via fiber optic link to an RZ5 processor (Tucker Davis Technologies).

For multiunit and single-unit analysis, signals were high-pass filtered off-line ( $300 \mathrm{~Hz} ; 48 \mathrm{~dB}$ /octave rolloff), and common average referencing was applied to each individual channel as described in the study by Ludwig et al. (2009). Spikes were extracted at 4-5 SDs above the background noise using the algorithm described by Quiroga et al. (2004). Events exceeding 20 SDs were rejected as artifacts. Extracted spike waveforms were peak aligned, hierarchically clustered, and sorted in principal component space using the open-source package UltraMegaSort 2000 for MATLAB (Hill et al., 2011). A cluster was defined as a single unit if it met the following criteria: (1) clear separation from neighboring clusters in principal component space; $(2) \leq 10 \%$ of spikes violating the refractory period; and (3) $\leq 5 \%$ spikes missing, as estimated from a Gaussian fit of the spike amplitude histogram (Caras and Sanes, 2017). Clusters that did not meet these criteria were considered multiunits. Because of the limited number of single units in our dataset (Table 2), we pooled single units and multiunits for all analyses reported here. The firing rate (in spikes per second) of each unit was calculated over a $1 \mathrm{~s}$ window for both modulated and unmodulated noise stimuli. Firing rates were converted into $d^{\prime}$ as follows:
Table 2. Breakdown of the number units (animals) recorded from each day of perceptual training

\begin{tabular}{|c|c|c|c|c|}
\hline & \multicolumn{2}{|l|}{ Adolescent } & \multicolumn{2}{|l|}{ Adult } \\
\hline & Single unit & Multiunit & Single unit & Multiunit \\
\hline Day 1 & $12(5)$ & $42(5)$ & $2(2)$ & 29 (4) \\
\hline Day 2 & $12(3)$ & 39 (6) & $3(1)$ & $32(4)$ \\
\hline Day 3 & $10(5)$ & $35(6)$ & $2(1)$ & $37(4)$ \\
\hline Day 4 & $7(3)$ & $33(5)$ & $0(0)$ & $33(4)$ \\
\hline Day 5 & $3(2)$ & $29(5)$ & $3(3)$ & $29(4)$ \\
\hline Day 6 & $7(2)$ & $27(3)$ & $1(1)$ & $28(3)$ \\
\hline Day 7 & $4(3)$ & $37(5)$ & $2(1)$ & $30(3)$ \\
\hline Subtotal & $55(5)$ & $242(6)$ & $13(3)$ & $218(4)$ \\
\hline Total & \multicolumn{2}{|c|}{$297(6)$} & \multicolumn{2}{|c|}{$231(4)$} \\
\hline
\end{tabular}

$$
d^{\prime}=\frac{2\left(\mu \mathrm{FR}_{\mathrm{AM}}-\mu \mathrm{FR}_{\mathrm{unmod}}\right)}{\sigma \mathrm{FR}_{\mathrm{AM}}+\sigma \mathrm{FR}_{\mathrm{unmod}}},
$$

where $\mu \mathrm{FR}_{\mathrm{AM}}$ and $\sigma \mathrm{FR}_{\mathrm{AM}}$ represent the mean and $\mathrm{SD}$ of the firing rate for a single modulation depth, and $\mu \mathrm{FR}_{\mathrm{unmod}}$ and $\sigma \mathrm{FR}_{\mathrm{unmod}}$ represent the mean and SD of the firing rate elicited by the unmodulated noise. Neural $d^{\prime}$ values were fit with a logistic function using a nonlinear leastsquares regression approach (MATLAB function nlinfit) and validated as previously described (Caras and Sanes, 2017). The neurometric threshold was defined as the AM depth at which the fit crossed $d^{\prime}=1$ with a positive-going slope. Units were considered responsive to AM noise if they generated a valid fit and either (1) elicited a valid threshold or (2) all $d^{\prime}$ values were $\geq 1$. For the latter case, the threshold was defined as the lowest AM depth presented during the training session.

Histology. At the end of all electrophysiology experiments, animals were administered an overdose of sodium pentobarbital $(150 \mathrm{mg} / \mathrm{kg}$, i.p.), and perfused with $0.01 \mathrm{M}$ PBS and $4 \%$ paraformaldehyde. In some animals, recording sites were marked via an electrolytic lesion by passing current $(7 \mathrm{~mA}, 10 \mathrm{~s})$ through one contact site immediately before perfusion. Brains were extracted, postfixed in $4 \%$ paraformaldehyde, and either (1) embedded in $6 \%$ agar and sectioned at $60 \mu \mathrm{m}$ on a benchtop vibratome (Leica); or (2) cryoprotected in $30 \%$ sucrose, embedded in a $10 \%$ gelatin $/ 30 \%$ sucrose mixture, postfixed in a $10 \%$ neutral buffered formalin $/ 20 \%$ sucrose solution for at least $48 \mathrm{~h}$, and sectioned at $40 \mu \mathrm{m}$ on a benchtop freezing microtome. Sections were mounted on gelatinsubbed slides, dried, and stained for Nissl. Bright-field images were acquired at $2 \times$ using a high-resolution slide scanner (VS-120, Olympus) or upright microscope (Revolve, Echo). Electrode tracks were reconstructed off-line and compared with a gerbil brain atlas (Radtke-Schuller et al., 2016) to confirm placement within core auditory cortex. Animals that participated in the behavioral-only experiments (Figs. 1, 2) were euthanized at the completion of the experiment via $\mathrm{CO}_{2}$ overdose.

\section{Experimental design and statistical analysis}

These experiments examined the effect of one between-subjects factor (age) and one within-subjects factor (time, quantified as either procedural trial number or perceptual training day) on behavioral and/or neural performance, as assessed by one or more dependent variables (e.g., $d^{\prime}$, trial number to reach training criterion, firing rate, and/or threshold). Both males $(n=24)$ and females $(n=28)$ were used. As no effect of sex was observed for our neural or behavioral measurements, we pooled data from males and females for all statistical analyses. For a detailed breakdown of the number of units collected from each animal on each day of the electrophysiological experiment, see Table 2.

Statistical analyses were performed using JMP Pro version 13.2.0 or 14.1.0 (SAS Institute) or MATLAB (MathWorks). Only responsive units (as defined above) were included in the electrophysiological analyses. No subjects were excluded from the study. Throughout this manuscript, $n$ refers to the number of animals and/or the number of multiunits or single units. Sample sizes for each analysis are presented in the text, figure, and/or figure legend. For normally distributed data (assessed using the Shapiro-Wilk test), mean values are reported or shown in figures, and parametric tests were used to assess statistical significance. For nonnormally distributed data, median values are reported or shown and 
A

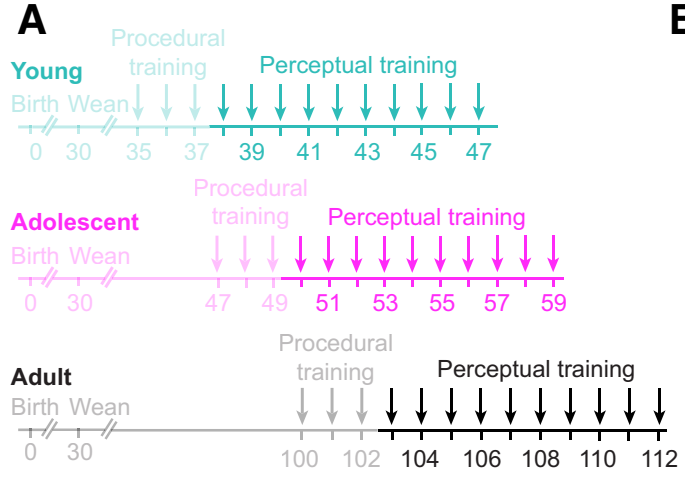

B

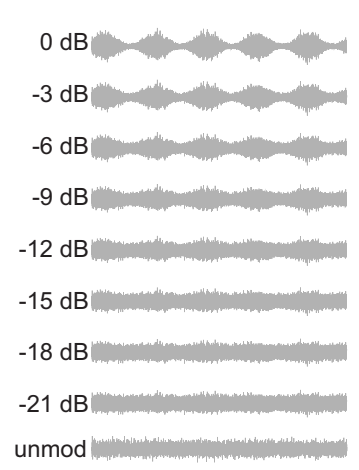

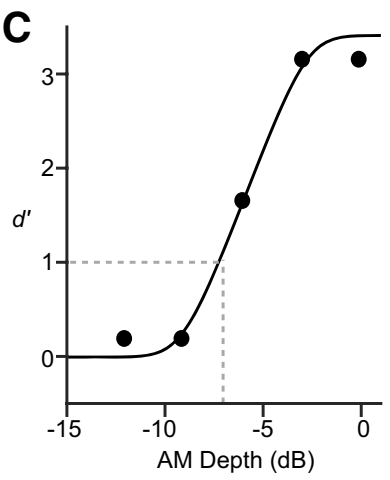

D

Day
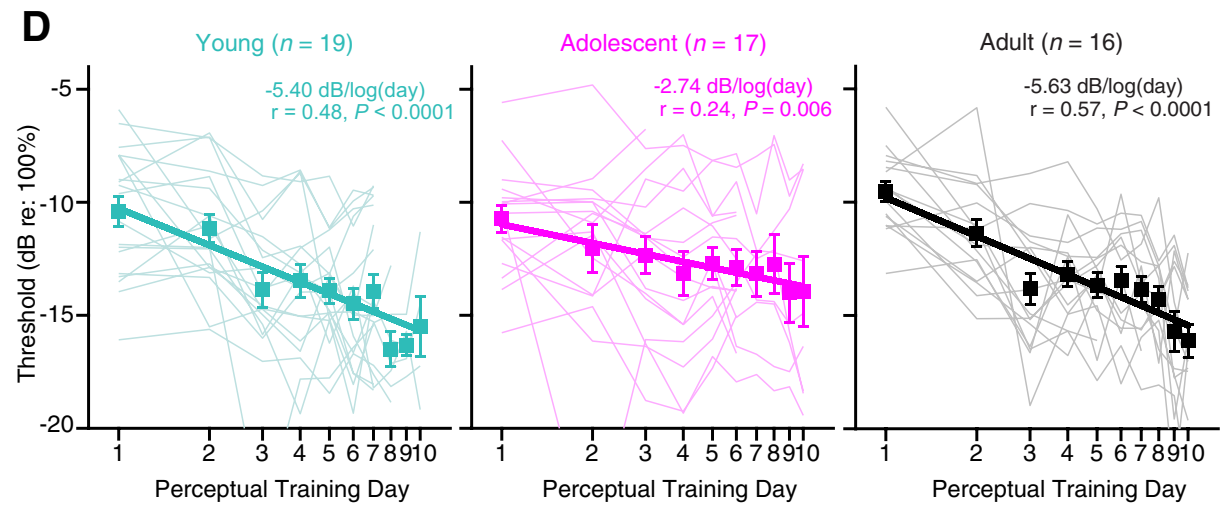

E

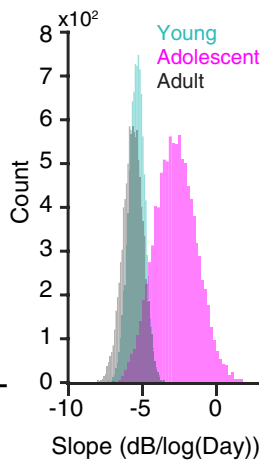

Figure 2. Perceptual learning is slower in adolescence. $A$, Experimental timeline for perceptual training. Ages depicted here are exact for young animals, and approximate for adolescent and adult animals (for details, see Materials and Methods). B, During perceptual training, warn trials consisted of AM noise across a wide range of depths. Depths are presented in decibels relative to $100 \%$, such that $0 \mathrm{~dB}$ is fully modulated noise. C, A representative psychometric function from the first day of perceptual training is plotted for one adolescent (subject ID 203447). Threshold was calculated as the AM depth at which the fitted function crossed $d^{\prime}=1$ (dashed line). $\boldsymbol{D}$, Perceptual learning trajectories are plotted for each age group as a function of the log of the perceptual training day. Individual trajectories are indicated by thin lines. Squares and error bars represent the mean \pm SEM values; thick straight lines represent the linear regressions. The slope, Pearson's $r$, and statistical significance for each fit is presented in the top right corner of each plot. The number of subjects in each group is indicated above each panel. Note that for visual clarity, the $y$-axes are truncated at $-20 \mathrm{~dB}$; however, four data points fell outside this limit: one young point (day 3,-24.2 dB), one adolescent point (day 2, $-25.6 \mathrm{~dB}$ ), and two adult points (days 9 and $10,-22.4$ and $-22.7 \mathrm{~dB}$ ). $\boldsymbol{E}$, Bootstrapped slope distributions are plotted for each age group. See text for statistical details. unmod, Unmodulated noise.

nonparametric tests were used. Data dispersion is reported either by plotting the full distribution of data or by showing SEs (for normally distributed data) or quartiles (for non-normally distributed data). Appropriate tests were used when data were found to violate assumptions of equal variance (as assessed using Levene's test). When data were found to violate assumptions of sphericity (as assessed using Mauchly's test), the Greenhouse-Geisser correction was applied. Statistical significance was assessed at an $\alpha$ level of 0.05 , and only two-tailed tests were used. When required, the $\alpha$ level was adjusted to correct for multiple comparisons using the Holm-Bonferroni method. Statistical details for each analysis can be found in the text of the Results section.

Data and code accessibility

All raw data, analysis code, and relevant descriptions can be found at https://nyu.box.com/v/caras-sanes-2019.

\section{Results}

\section{The rate and magnitude of procedural learning are similar} across age

To determine whether procedural learning (Ortiz and Wright, 2009) changes during development, we trained and tested 52 gerbils on an auditory detection task in which subjects were required to withdraw from a lickspout when a safe stimulus (unmodulated broadband noise) smoothly transitioned to a warn stimulus ( $0 \mathrm{~dB}$ at $100 \%$ AM noise; Fig. $1 A, B)$. Animals began procedural training at the following three different ages (Fig. $1 C$ ): P38 ("young," $n=19$ ), between P41 and P49 ("adolescent," $n=$ 17), and P96 or later ("adult," $n=16$ ). As illustrated in Figure $1 D$, behavioral sensitivity $\left(d^{\prime}\right)$ improved with practice (mixed-model ANOVA, $\left.F_{(71,3479)}=63.00, p<0.0001\right)$. However, there was no effect of age on the overall rate or magnitude of procedural learning $\left(F_{(2,49)}=0.645, p=0.529\right)$. Thus, all age groups required a similar number of trials to reach the performance criterion to begin psychometric testing ( $d^{\prime} \geq 1.5$; Kruskal-Wallis test by ranks, $\mathrm{H}=3.23, p=0.199$; Fig. $1 E$ ) and achieved comparable high maximum $d^{\prime}$ values (Kruskal-Wallis test by ranks, $\mathrm{H}=2.91$, $p=0.233$; Fig. $1 F$ ). Improved behavioral performance was attributable to both a significant increase in hit rate (mixed-model ANOVA, $\left.F_{(72,3528)}=63.13, p<0.0001\right)$ and a decrease in false alarm rate $\left(F_{(71,3479)}=3.55, p<0.0001\right)$. These results indicate that developmental age is not associated with the ability of animals to learn an AM detection procedure.

\section{Perceptual learning is slower in adolescent animals}

Following procedural acquisition, animals began perceptual training (Fig. 2A), during which they were challenged with a range of AM depths (Fig. $2 B$ ). The data from each training session were fit to generate a psychometric function, and threshold was defined as the AM depth at which the fitted function crossed $d^{\prime}=$ 1 (Fig. $2 C$ ). On the first day of perceptual training, all age groups displayed similar thresholds (one-way ANOVA: $F_{(2,49)}=1.12$, $p=0.333$; Fig. $2 D$ ), with a trend toward unequal variances (Levene's test: $\left.F_{(2,49)}=2.95, p=0.062\right)$. Between-subject variability was greatest in young animals, and gradually decreased with age 
(young, SD 2.85; adolescent, SD 2.43; adult, SD 1.77). This finding is consistent with previous reports of increased threshold variability in developing nonhumans (Sarro and Sanes, 2010, 2011; Green et al., 2016) and children (Walker et al., 2006; Halliday et al., 2008; Moore et al., 2008, 2011; Huyck and Wright, 2011, 2013).

In general, individual animals improved with practice, such that thresholds decreased across training sessions. However, while the majority of young and adult animals showed clear improvement, many adolescents showed little to no change or showed erratic learning trajectories (Fig. 2D). At the group level, this age-related difference in perceptual learning was significant [ANCOVA, age $\times \log$ (day) interaction, indicating unequal slopes; $\left.F_{(2,428)}=3.80, p=0.023\right]$. Young animals improved at a rate of $-5.40 \mathrm{~dB} / \log$ (day) (Pearson's $r=0.48, p<0.0001$ ), similar to adults $[-5.63 \mathrm{~dB} / \log ($ day $) ; r=0.57, p<0.0001]$. In contrast, adolescents improved at half that rate $[-2.74 \mathrm{~dB} / \log$ (day); $r=0.24, p=0.006]$. Post hoc pairwise analyses confirmed that adolescents learned significantly more slowly than individuals in either the young $(t=2.27, p=0.024)$ or adult $(t=2.52, p=$ 0.012 ) groups, whereas young and adult animals learned at similar rates $(t=0.20, p=0.840)$.

To obtain a better estimate of the regression slope for each age group, we performed a bootstrap analysis in which a random sample of subjects was drawn from each age group with replacement, the thresholds for each of these new samples were fit with a linear regression against $\log ($ day $)$, and the slope of each regression was calculated. This process was repeated 10,000 times to generate a distribution of slope values for each age group. As shown in Figure 2E, the adolescent distribution is shifted toward shallower values. Welch's ANOVA (which accounts for unequal variances across groups) revealed a significant effect of age $\left(F_{(2,18382)}=17968.19, p<0.0001\right)$. A Tukey's HSD post hoc analysis revealed that the average adolescent slope $[-2.73 \mathrm{~dB} /$ $\log ($ day $)]$ was approximately half as steep as that of the adult $(p<$ $0.0001)$ or young animals $(p<0.0001)$.

Slower adolescent learning could not be explained by insufficient practice, as adults and adolescents completed a similar number of warn trials per session [median (range); adolescent, 73 (24-98); adult, $67(50-124)]$. Only the young group differed, performing significantly more warn trials per session [82 (69105); Kruskal-Wallis test by ranks, $\mathrm{H}=9.47, p=0.009$ ] likely as a result of a decreased ability to retain water between sessions. Similarly, the results are unlikely to be attributed to differences in the sensitivity to the aversive shock, as false alarm rates were equivalently low for all groups [median (range); young, 3\% (19\%); adolescent, 3\% (0-7\%); adult: 4\% (0-9\%); Kruskal-Wallis test by ranks, $\mathrm{H}=0.299, p=0.861$ ]. In addition, we calculated the response bias (as measured by the signal detection metric $C$, which corresponds to the location of the decision criterion) for 2 stimulus values presented throughout training $(-9$ and $-12 \mathrm{~dB})$. A mixed-model ANOVA revealed that there was no effect of age on $C\left(-9 \mathrm{~dB}: F_{(2,19)}=0.997, p=0.388 ;-12 \mathrm{~dB}: F_{(2,22)}=1.27\right.$, $p=0.300)$ and no age $\times$ day interaction $\left(-9 \mathrm{~dB}: F_{(18,171)}=1.32\right.$, $\left.p=0.182 ;-12 \mathrm{~dB}: F_{(18,198)}=0.670, p=0.839\right)$. Together, these findings suggest that it is unlikely that nontask factors affect behavioral strategy differently at each age.

To determine whether poor adolescent performance was attributable to any specific data points exerting an oversized influence on the adolescent regression, we calculated the Mahalanobis distance (MD) for each data point in the adolescent group. The $\mathrm{MD}$, a test for multivariate outliers, is a measure of the distance between a single data point and the overall bivariate [i.e., log- (day) $\times$ threshold] distribution, and takes into account the shape of the correlation structure (e.g., spherical, ellipsoidal). A data point with a greater MD value than the rest of the sample is suggestive of an outlier. One data point, which corresponded to a threshold of $-25.6 \mathrm{~dB}$ from a single adolescent animal on the second day of perceptual training was identified as a likely outlier. When this point was removed from the adolescent dataset, the general finding still held true [ANCOVA age $\times \log$ (day) interaction indicating unequal slopes: $\left.F_{(2,427)}=3.12, p=0.0454\right]$. None of the other adolescent data points deviated from the MD distribution and thus were not considered outliers.

Together, these findings indicate that perceptual learning skills follow a nonlinear developmental trajectory, consistent with reports that learning capabilities are diminished during adolescence (Huyck and Wright, 2011, 2013; van der Schaaf et al., 2011; Pattwell et al., 2012; Lukács and Kemény, 2015; Knoll et al., 2016).

\section{Adolescents display reduced cortical activity compared with adults}

Our behavioral results suggest that the neural mechanisms supporting perceptual learning are diminished during adolescence. We explored this possibility by implanting a subset of adolescent $(n=6)$ and adult $(n=4)$ animals with 16-channel electrode arrays to wirelessly record single-unit and multiunit activity from left core auditory cortex as animals underwent perceptual training (Fig. $3 A, B$ ). Note that we limited this analysis to the first $7 \mathrm{~d}$ of perceptual training because we only had neural data from two adults on days $8-10$. We recorded from a total of $528 \mathrm{AM}$ responsive units (adolescent, $n=297$; adult, $n=231$ ) across $7 \mathrm{~d}$ (for full details, see Table 2).

We first examined whether adolescent and adult auditory cortical neurons differed in their firing properties at the start of perceptual training. We limited this initial analysis to multiunit data collected on the first day of perceptual training (adolescent, $n=42$ of 297 units; adult, $n=29$ of 231 units; Table 2) because single-unit and multiunit firing rates often differ by an order of magnitude. As illustrated for two representative units in Figure 3, $C$ and $D$, AM noise elicited robust, time-locked responses from both age groups. However, the distribution of maximum-evoked multiunit spike rates was shifted toward lower values in adolescents $($ median $=46.5 \mathrm{~Hz}$; range $=4.27-101 \mathrm{~Hz})$ compared with adults (median $=65.5 \mathrm{~Hz}$; range $=6.83-185 \mathrm{~Hz}$; Welch's $t$ test, $t_{(38.66)}=2.05, p=0.048$; Fig. $3 E$ ). Similarly, adolescent multiunit responses were lower across the range of AM depths presented, including unmodulated noise (mixed-model ANOVA, $F_{(1,69)}=$ $7.14, p=0.0094$; Fig. $3 F$ ). Despite these age-related differences in firing rates, the relative change between unmodulated and AMevoked activity was similar in both groups, such that the average neural $d^{\prime}$ values were nearly identical (mixed-model ANOVA, $F_{(1,69)}=0.194, p=0.661 ;$ Fig. $\left.3 G\right)$. The tight correspondence in neurometric sensitivity translated to similar multiunit thresholds across age (Wilcoxon rank-sum test, $Z=-0.310, p=0.756$; Fig. $3 H$ ). Together, these results indicate that, despite diminished spiking activity, adolescents and adults begin perceptual training with similar cortical AM detection capabilities.

\section{Adolescent cortical neurons display slower improvements in sensitivity compared with adults}

Our initial behavioral study revealed that adolescents display slower and more erratic perceptual learning trajectories than adults. Similar findings were observed with the smaller sample of animals used for electrophysiological recordings. Figure $4 \mathrm{~A}$ 


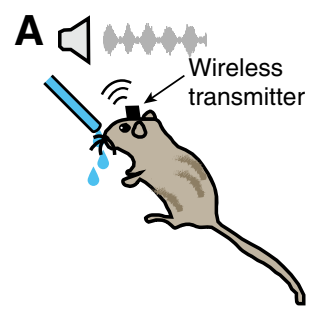

D Adolescent

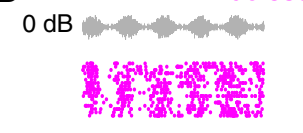

$-3 \mathrm{~dB}$
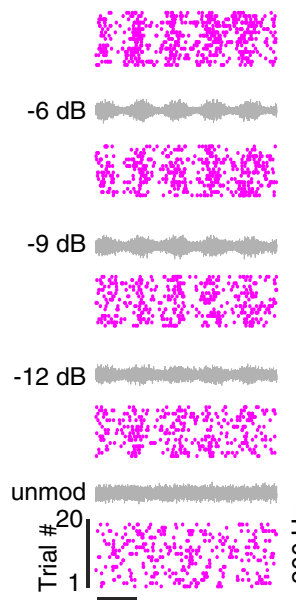

$\overline{200} \mathrm{msec}$

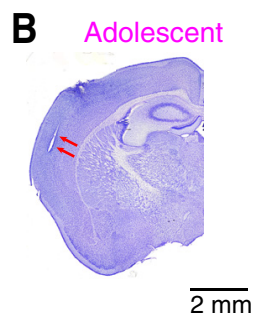

$\overline{2 \mathrm{~mm}}$

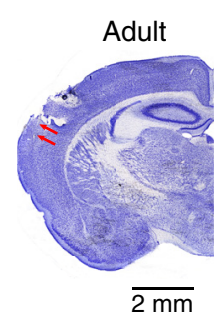

Adult
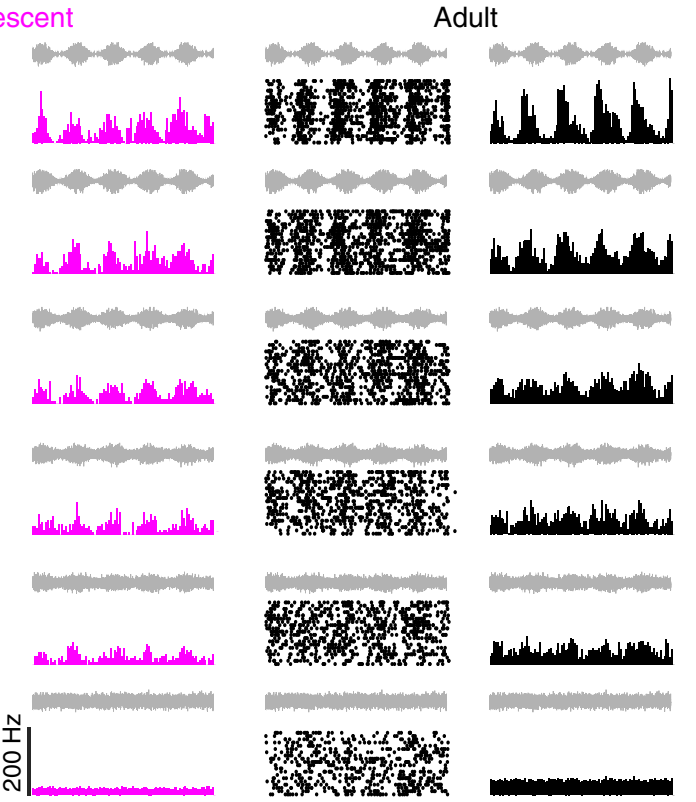

B Representative raw voltage traces are plotted for an adolescent (subject ID 201700, channel 11) and an adult (subject ID 217821, channel 2), along with the warn stimulus envelopes (adolescent, -6 dB; adult, - 12 $\mathrm{dB}$; gray). Both recordings were obtained on day 5 of perceptual training. D, Raster plots (left) and poststimulus time histograms (right) are plotted for two different multiunits (adolescent subject ID 201700, channel 1; adult subject ID 221955, channel 15). Plots are arranged in order of decreasing AM depth. Stimulus envelopes are pictured in gray. The activity depicted here, and for all subsequent panels in this figure, was recorded on the first day of perceptual training. $\boldsymbol{E}$, The distribution of maximum firing rates are plotted for all adolescent and adult multiunits. For this, and all subsequent panels in this figure, the number of units (animals) are as follows: adolescent, 42 (5); adult, 29 (4). F, Adolescent and adult firing rates are plotted as a function of AM depth. Data are the mean \pm SEM. G, Adolescent and adult firing rates were transformed into $\boldsymbol{d}^{\prime}$ values and plotted as a function of AM depth. Data are the mean \pm SEM. $\boldsymbol{H}$, Thresholds for individual multiunits or single units (circles) are plotted as a function of age group for the first day of perceptual training. Horizontal lines represent medians, boxes represent the 25 th and 75 th percentiles, and whiskers represent the 5 th and 95 th percentiles. unmod, Unmodulated noise; Max, maximum; sp/s, Spikes per second.

shows the change in threshold relative to the first day of perceptual training for each implanted adolescent (Fig. 4A, pink lines) and the full range of improvement for implanted adults (Fig. 4A, gray shading). The majority of adolescents (five of six animals) showed a delay in improvement, with some animals showing little benefit of training, or a transient improvement followed by worsening. To determine whether neural sensitivity also improved with training, multiunit and single-unit thresholds were averaged within individual animals on each training day. As shown in Figure $4 B$, the rate of auditory cortical improvement was slower than adults in four of six adolescents. Behavioral and neural trajectories were well matched within individual animals, as can be seen by comparing lines with identical styles across Figure $4 A$ and $B$, and by examining Figure $4, C$ and $D$, which illustrates that neural and behavioral thresholds were highly correlated both within the adolescent (Pearson's $r=0.68, p<$ $0.0001)$ and adult $(r=0.74, p<0.0001)$ animals. At the group level, average neural thresholds were significantly slower to improve in adolescents than in adults [ANCOVA age $\times \log ($ day $)$ interaction indicating unequal slopes, $F_{(1,10)}=11.71, p=0.0065$; Fig. $4 E]$. While adult cortical thresholds decreased at a rate of
$-7.0 \mathrm{~dB} / \log$ (day) (Pearson's $r=0.98, p=0.0001$ ), adolescent neurons improved at less than half that rate $[-2.9 \mathrm{~dB} / \log ($ day); $r=0.79, p=0.0334]$, such that by day 7 , neural thresholds were significantly higher in adolescents than adults (adolescent, $-9.18 \pm 0.52 \mathrm{~dB}$; adult, $-12.1 \pm 0.44 \mathrm{~dB}$; Student's $t$ test, $t_{(71)}=$ $-3.16, p=0.0023)$. These findings are not likely to be explained by differences in practice, as the number of warn trials did not differ significantly during training (means $\pm \mathrm{SD}$; adolescent, $60 \pm 20$ trials/session; adult, $81 \pm 37$ trials/session; mixed-model ANOVA, $\left.F_{(1,7)}=1.50, p=0.260\right)$. Together, these findings suggest that training generates smaller improvements in auditory cortical sensitivity in adolescents, leading to a slower rate of perceptual learning.

\section{Adolescent cortical neurons exhibit variable responses to perceptual training}

There are two possible explanations for the weak cortical improvement displayed by adolescents. First, similar to what we have reported previously for adult animals (Caras and Sanes, 2017), the entire population of adolescent neural thresholds may gradually improve throughout perceptual training, but the mag- 

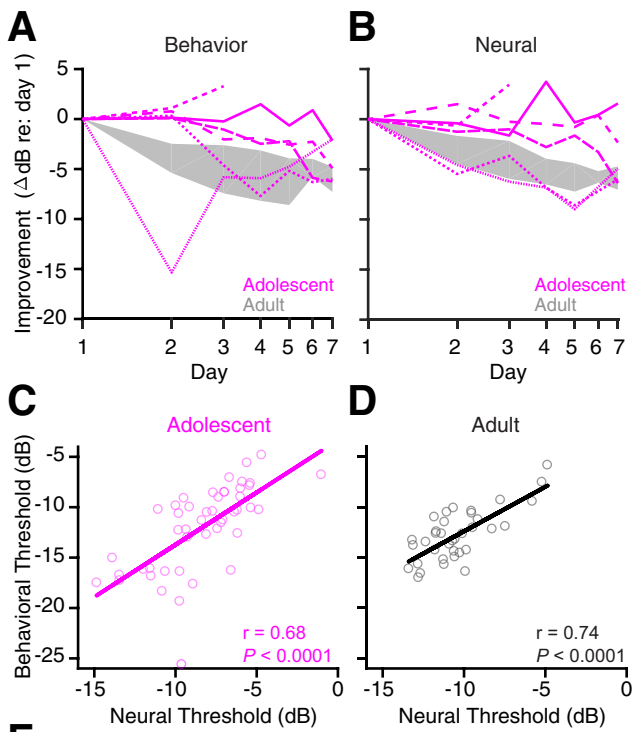

E

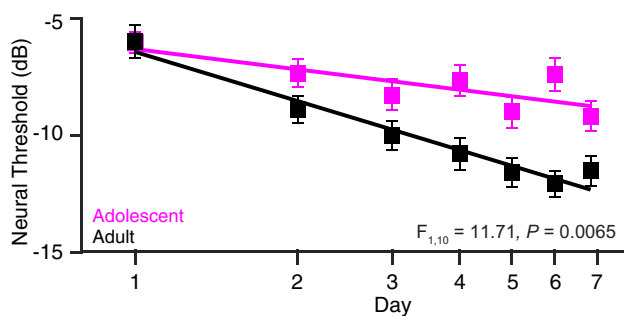

Figure 4. Adolescents display slower improvements in cortical sensitivity compared with adults. $A$, Behavioral improvement (i.e., the change in threshold relative to day 1 ) is plotted for each individual adolescent animal implanted with a chronic electrode (pink lines) as a function of the perceptual training day. The gray shaded region represents the full range of behavioral improvement in adult implanted animals. $\boldsymbol{B}$, Cortical thresholds were averaged across individual multiunits and single units within an animal. Neural improvement was calculated as the change in the average neural threshold relative to day 1. Data from the same animal are depicted with identical line styles in $\boldsymbol{A}$ and $\boldsymbol{B}$. The gray shaded region represents the full range of adult neural improvement. $\boldsymbol{C}$, Each circle represents the average neural threshold recorded in a given animal during a single training session, plotted against the behavioral threshold of the animal from that same training session. Neural and behavioral thresholds were highly correlated within adolescent animals. $\boldsymbol{D}$, Same as $\boldsymbol{C}$, but for adults. $\boldsymbol{E}$, Neural thresholds were averaged across all animals in a given age group and plotted as a function of the log of the perceptual training day. For the number of units and subjects recorded from on each day, see Table 2.

nitude of change for each unit may be comparatively smaller than in adults. Alternatively, the adolescent neural population may be variable in its response to training, with some individual units displaying improved sensitivity and others displaying stable or degraded sensitivity, such that the average improvement at the group level is relatively small. To explore these two possibilities, we calculated thresholds for individual multiunits (and a smaller number of single units) that we were able to monitor across multiple training days. As shown in Figure $5 A$ (right), nearly all adult units had lower (better) thresholds on day 2 of perceptual training compared with day 1 , as indicated by the fact that the majority of points fall above the unity line. This threshold shift was significant (mean \pm SEM; day $1,-6.76 \pm 0.97 \mathrm{~dB}$; vs day $2,-9.01 \pm$ $0.76 \mathrm{~dB}$; Student's paired $t$ test, $\left.t_{(15)}=-5.50, p<0.0001\right)$. In contrast, the response of individual adolescent units was more variable (Fig. 5A, left), such that the overall threshold difference between day 1 and day 2 was negligible (mean \pm SEM; day 1 , $-6.69 \pm 0.60 \mathrm{~dB}$; vs day $2,-7.83 \pm 0.58 \mathrm{~dB} ; t_{(32)}=-1.83, p=$ 0.076 ). Similar results were observed as training continued (Fig. $5 B-D)$. For example, while individual adolescent units were
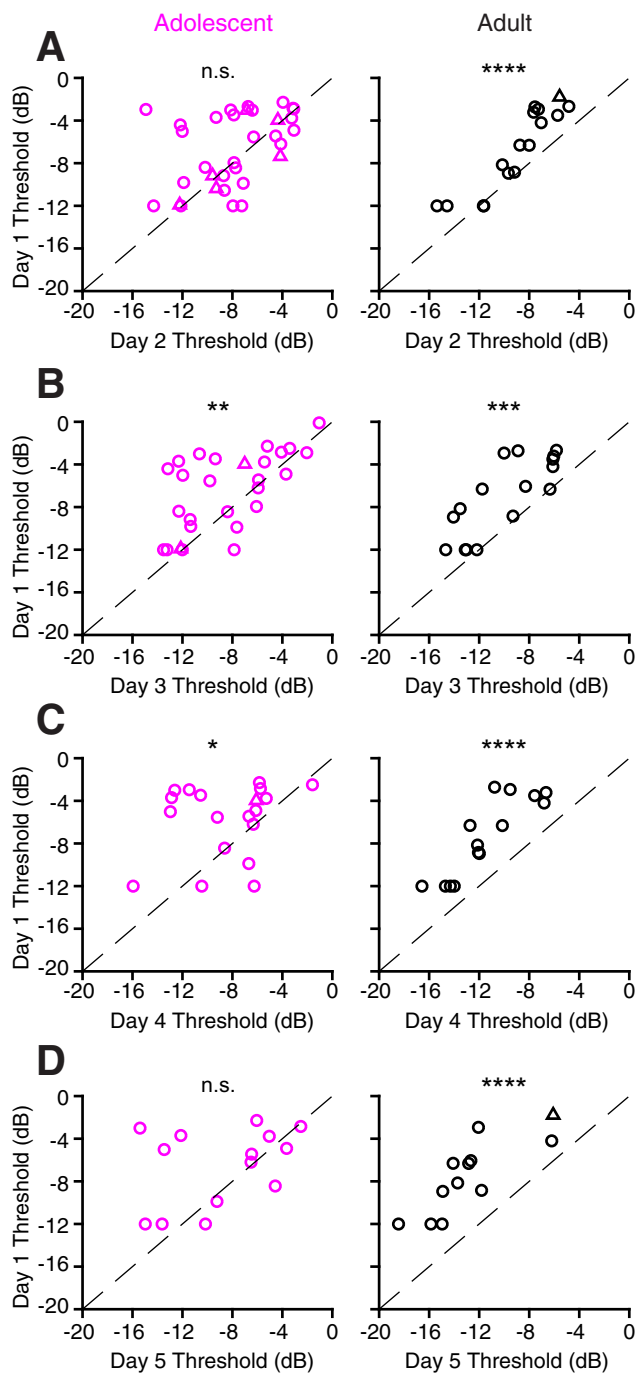

Figure 5. Adolescent neurons exhibit variable responses to perceptual training. $\boldsymbol{A}$, Multiunit (circles) and single-unit (triangles) thresholds from the first day of perceptual training are plotted against thresholds from the second day of perceptual training. Only units that were successfully monitored across multiple consecutive training days were included in this and all subsequent panels in this figure. Data points falling above the line of unity (dashed line) are units that improved from day 1 to day 2 ; data points falling below this line are units that worsened. The number of units (animals) contributing to this dataset are as follows: adolescent, 33 (4); adult, 16 (2). $\boldsymbol{B}-\boldsymbol{D}$, Same as $\boldsymbol{A}$, but for comparisons of day 1 with day 3 [ $\boldsymbol{B}$; adolescent, 27 (4); adult, 16 (2)], day 4 [C; adolescent, 19 (2); adult, 14 (2)], and day 5 [D; adolescent, 14 (2); adult, $12(2)]$. The panels depicting adult data in this figure were originally published in the study by Caras and Sanes (2017). ${ }^{*} p<0.05,{ }^{* *} p<0.01,{ }^{* * *} p<0.001,{ }^{* * * *} p<0.0001$. n.S., not significant.

often found to exhibit stable or even poorer thresholds in later sessions, by day 4 all adult units had improved. As a result of this increased neural variability, the adolescent population consistently exhibited smaller average improvements (as measured using Cohen's $d$ estimate of effect size) than adults (day 1 vs day 3: adolescent, $t_{(26)}=-3.02, p=0.0057, d=0.52$; adult, $t_{(15)}=-5.15, p=0.0001, d=0.84$; day 1 vs day 4 : adolescent, $t_{(18)}=-2.69, p=0.0148, d=0.76$; adult, $t_{(13)}=$ $-8.33, p<0.0001, d=1.2$; day 1 vs day 5 : adolescent, $t_{(13)}=$ $-1.84, p=0.0893, d=0.55$; adult, $t_{(11)}=-8.55, p<0.0001$, $d=1.5)$. These findings suggest that variable neuronal responses to training across the adolescent population contributes to slower average behavioral threshold improvements. 

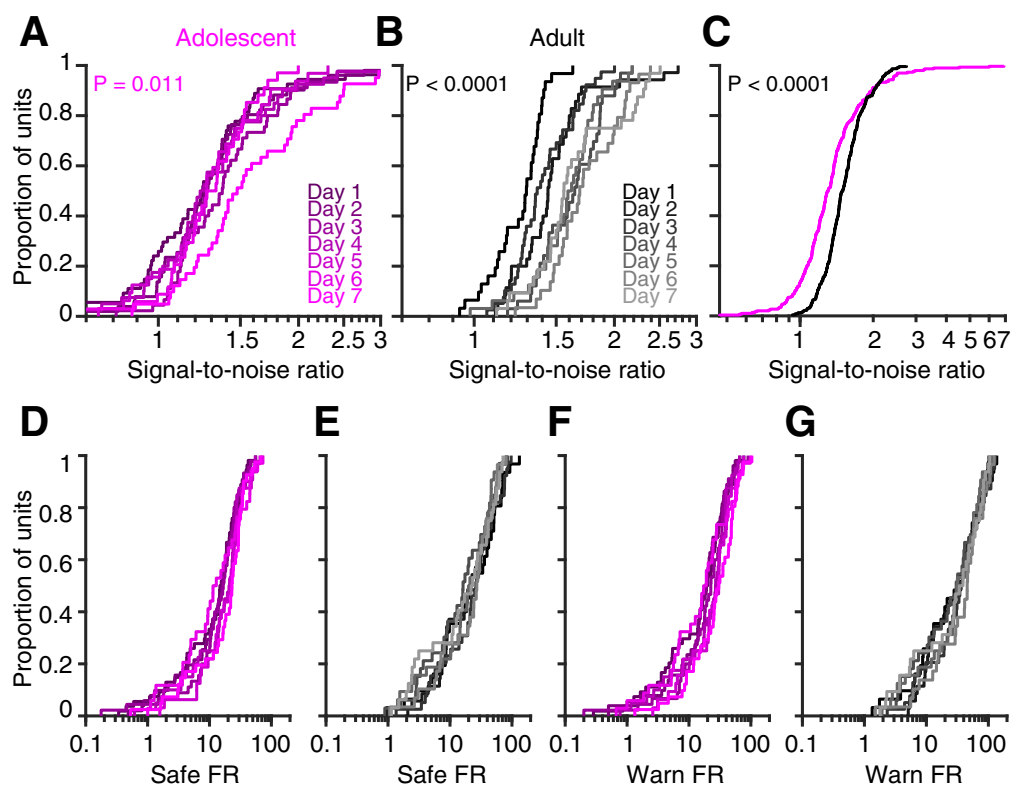

E
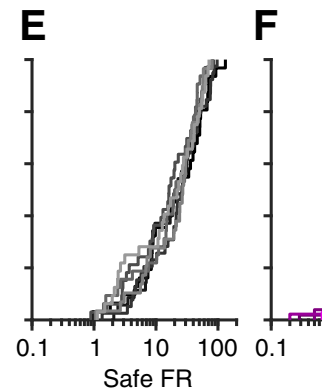

G

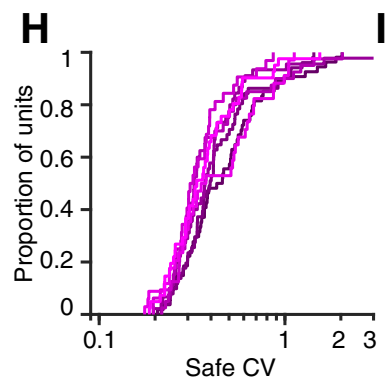

I
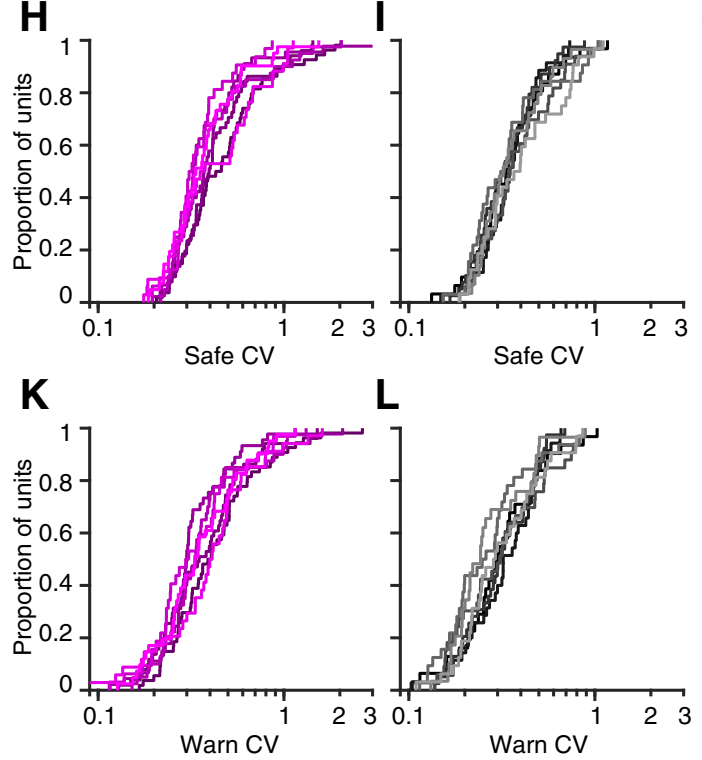

L

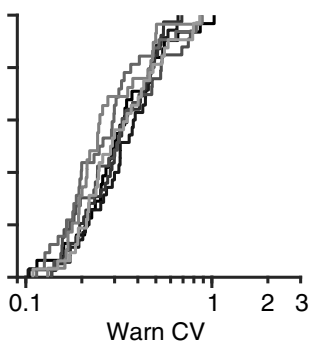

\section{M}

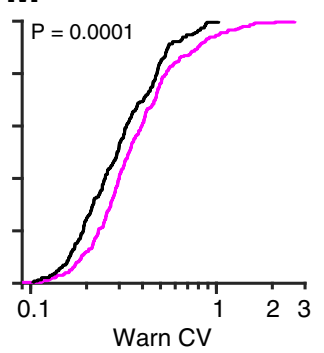

Figure 6. Adolescent neurons display increased trial-to-trial variability and poorer signal-to-noise ratios. $A, B$, Signal-to-noise ratios were calculated for individual multiunits and single units by dividing the mean firing rate evoked by a moderate warn stimulus ( $-9 \mathrm{~dB} A M)$ by the mean firing rate evoked by the safe (unmodulated noise) stimulus. A value of 1 indicates that the unit responded identically to safe and warn stimuli; values $>1$ indicate greater separation between the two responses. The cumulative distribution of signal-to-noise ratios for the adolescent $(\boldsymbol{A})$ and the adult $(\boldsymbol{B})$ neural population is plotted for each perceptual training day. $C$, The distribution of signal-to-noise ratios is plotted for each age group, collapsed across training sessions. $\mathbf{D}-\mathbf{G}$, Cumulative distributions for safe-evoked $(\boldsymbol{D}, \boldsymbol{E})$ and warn-evoked $(\boldsymbol{F}, \boldsymbol{G})$ firing rates $(F R s)$ in adolescents $(\boldsymbol{D}, \boldsymbol{F})$ and adults $(\boldsymbol{E}, \boldsymbol{G})$ are plotted for each day of perceptual training. $\boldsymbol{H}, \boldsymbol{I}$, Cumulative distributions for safe-evoked $(V$ values for the adolescent $(\boldsymbol{H})$ and adult (I) neural population are plotted for each day of perceptual training. J, The safe-evoked $C V$ is plotted for each age group, collapsed across training sessions. $\boldsymbol{K}, \boldsymbol{L}$, Cumulative distributions for warn-evoked $C V$ values for the adolescent $(\boldsymbol{K})$ and adult $(\boldsymbol{L})$ neural populations are plotted for each day of perceptual training. $M$, The warn-evoked $C V$ is plotted for each age group, collapsed across training sessions. For the number of units and animals recorded from each day and represented in $A, B, D-I, K, L$, see Table 2 . For $\boldsymbol{C}, \boldsymbol{J}$, and $\boldsymbol{M}$, the number of units (animals) contributing to the analysis are as follows: adolescent, 297 (6); adult, 231 (4). $B, E, G, I$, and $\boldsymbol{L}$ were originally published in a similar form in the study by Caras and Sanes (2017).

\section{Adolescent cortical neurons display increased trial-to-trial variability and poorer signal-to-noise ratios}

Our previous analysis of the adult data presented here (Caras and Sanes, 2017) found that training enhanced neural sensitivity by increasing the separability of warn-evoked and safe-evoked responses, effectively enhancing the signal-to-noise ratio. Therefore, we asked whether a similar process occurred in adolescents, or whether training improved adolescent neural sensitivity via an alternative approach, such as reducing trial-to-trial response variability (Dosher and Lu, 1998, 1999; Jones et al., 2013; Amitay et al., 2014; von Trapp et al., 2016). To address this issue, we first calculated the signal-tonoise ratio (mean warn-evoked firing rate/mean safe-evoked firing rate) for each individual multiunit and single unit on each training day. Because stimulus values were adjusted throughout training to bracket behavioral thresholds (see Materials and Methods), we limited this analysis to one moderate AM depth $(-9 \mathrm{~dB})$ that was presented in all sessions. Signalto-noise ratios gradually improved with practice for both adolescents (KruskalWallis test by ranks, $\mathrm{H}=16.62, p=0.011$; Fig. $6 A)$ and adults $(\mathrm{H}=65.77, p<$ 0.0001 ; Fig. $6 B$ ). However, the degree of improvement was weaker in adolescents, leading to a smaller signal-to-noise ratio overall when collapsed across days (Wilcoxon rank-sum test, $Z=6.54, p<$ 0.0001 ; Fig. $6 C$ ). Consistent with our previous report (Caras and Sanes, 2017), training induced either marginal or no systematic change in the average population firing rate (safe-evoked: adolescent, $\mathrm{H}=8.06, p=0.234$; Fig. $6 D$; adult, $\mathrm{H}=$ 2.90, $p=0.821$; Fig. $6 E$; warn-evoked: adolescent, $\mathrm{H}=13.14, p=0.041$, Fig. $6 F$; adult, $\mathrm{H}=2.22, p=0.898$, Fig. $6 G$ ).

To assess trial-to-trial response variability, we calculated the coefficient of variation $(\mathrm{CV})$ for each unit. Adolescents displayed steady safe-evoked (KruskalWallis test by ranks, $\mathrm{H}=11.62, p=0.071$; Fig. $6 H)$ and warn-evoked $(\mathrm{H}=8.63, p=$ 0.196; Fig. $6 \mathrm{~K}) \mathrm{CV}$ values across days, as did adults (safe: $\mathrm{H}=3.61, p=0.729$; Fig. $6 I$; warn: $\mathrm{H}=7.24, p=0.299$; Fig. $6 L)$. However, both safe $(Z=-2.70, p=$ 0.0070; Fig. $6 J$ ) and warn (Wilcoxon rank-sum test, $Z=-3.82, p=0.0001$; Fig. 6M) CV values were consistently higher in adolescents than in adults when collapsed across days. Collectively, these findings suggest that adolescent neural sensitivity is limited by both a poorer ability to separate signal from noise and a greater overall trial-by-trial response variability.

\section{Discussion}

Experience-dependent plasticity plays a significant role in shaping the developing nervous system, and is thought to support skill acquisition during childhood. However, the capacity to learn is not fully developed at birth, and instead emerges slowly through the juvenile period (Moye and Rudy, 1987; Camp and Rudy, 1988; Overman, 1990). Here, we found that perceptual learning of amplitude modulation detection follows a nonlinear developmental trajectory, such 
that learning skills are transiently diminished during adolescence. By monitoring auditory cortical activity of developing and adult gerbils as they trained on an identical task, we found evidence that adolescent learning is associated with relatively weak trainingbased improvements in auditory cortical sensitivity. This limited neural plasticity was explained, in part, by a greater similarity between signal-evoked and noise-evoked neural responses, and by higher trial-to-trial response variability. Below, we discuss these findings in the context of the broader development and learning literature.

\section{Development of skill learning}

Auditory perceptual learning follows a prolonged maturational time course. Practice-based improvements on both temporal interval discrimination (Huyck and Wright, 2011) and backward masked tone detection (Huyck and Wright, 2013) are slower in adolescents than in adults. Similarly, in both rat (Dean et al., 1990; Friedman et al., 2004) and gerbil (Green et al., 2016), perceptual learning on a gap detection task improves systematically as animals mature. However, some forms of learning decline with development. Songbirds, for example, learn to imitate the vocalizations of their tutor and are often capable of copying sounds that do not exist in their species-specific repertoire (Baptista and Morton, 1981; Woolley et al., 2010). In many species, this capacity for vocal learning is restricted to a specific early developmentally sensitive period (Marler and Tamura, 1964; Clayton, 1987). In contrast, some sensory and cognitive abilities are only temporarily diminished during adolescence (Pattwell et al., 2013; for review, see King et al., 2013, 2014; Hartley and Somerville, 2015). For example, adolescent mice and humans display slower rates of extinction learning for a previously conditioned fear response compared with younger juveniles or mature adults (Pattwell et al., 2012). A similar nonlinear developmental trajectory has been described for facial recognition (Carey et al., 1980), decisionmaking (Cauffman et al., 2010; Somerville et al., 2010, 2011; Decker et al., 2016), memory retention (McCallum et al., 2010), memory retrieval (Pattwell et al., 2011), reversal learning (van der Schaaf et al., 2011), and experiential learning (Decker et al., 2015; Lukács and Kemény, 2015). Thus, the development of learning does not follow a single trajectory, and is instead specific to a particular percept, task, or cognitive process. Our behavioral findings are broadly consistent with these studies. Auditory perceptual learning followed a nonlinear developmental time course, temporarily worsening during adolescence (Fig. 2).

\section{Increased behavioral variability during development}

Increased behavioral variability is a hallmark of auditory development and is well documented for both sensory thresholds (Allen and Wightman, 1994; Hartley et al., 2000; Walker et al., 2006; Moore et al., 2008, 2011; Sarro and Sanes, 2010) and perceptual learning trajectories (Halliday et al., 2008; Sarro and Sanes, 2010; Huyck and Wright, 2011, 2013; Green et al., 2016). Consistent with these findings, both young and adolescent animals displayed a trend toward greater between-subject variance of AM detection thresholds on the first day of perceptual training. Similarly, the behavioral thresholds of individual adolescents in our study were often quite variable across training and, in some cases, actually worsened. These findings highlight the fact that naive sensitivity does not always predict future performance, particularly during development. Similar results, including reports of traininginduced perceptual degradation, have been observed in children and animals training on a variety of auditory perceptual tasks
(Moore et al., 2008; Huyck and Wright 2011, 2013; Green et al., 2016).

Several theories have been advanced to explain this variability. One possibility is that individuals of the same chronological age may be at different maturational stages during training or testing. In fact, there is high between-subject variability of the extent, timing, and rate of maturation (Lloyd et al., 2014), particularly with regard to the adolescent growth spurt (Sanders et al., 2017) and puberty (Marshall and Tanner, 1969). Between-subject differences in sex-steroid hormone levels during adolescence are likely to contribute to these findings, as they directly impact auditory function (for review, see Sisneros, 2009; Caras, 2013) and, more generally, may contribute to the variable phases of brain development observed across juveniles at a given age (Brown, 2017). Therefore, our observation that adult-like learning is observed in some immature subjects (Fig. 2D) is consistent with previous behavioral findings (Halliday et al., 2008; Sarro and Sanes, 2010; Huyck and Wright, 2011; Green et al., 2016).

\section{Neural mechanisms contributing to poor adolescent perceptual learning}

In adult auditory cortex, training significantly improved the signal (mean warn-evoked firing rate) to noise (mean safe-evoked firing rate) ratio of individual multiunits and single units. In contrast, training had far less of an impact on adolescent units, such that signal-to-noise ratios remained steady, or showed limited improvement with practice. When collapsed across days, signal-to-noise ratios were significantly smaller in adolescents than in adults. In addition, adolescent neural responses were more variable across trials. Increased neural variability not only limits sensory performance (Green and Swets, 1966; Buss et al., 2006, 2009), but may impair behavioral improvement by generating an unstable and unpredictable percept (Amitay et al., 2014). Together, these results support the notion that adolescent learning is limited by both a smaller difference between the discharge rates evoked during warn and safe trials and greater internal noise.

While young and adult animals learn at similar rates, it does not necessarily mean that their underlying neural plasticity mechanisms are identical. In fact, animals of different ages might be using different neural strategies to improve their perceptual performance. For example, one possibility is that young animals rely more on plasticity in decision-making, such that the outputs of auditory cortex are preferentially reweighted during training (Dosher and Lu, 1998, 1999; Petrov et al., 2005; Law and Gold, 2008; Kahnt et al., 2011), while adults rely more on plasticity in sensory representations. Adolescence may represent a transition period during which neither strategy is optimally used, leading to more variable learning abilities. Recordings from young animals and from areas thought to be involved in decision-making, such as posterior parietal cortex (Lyamzin and Benucci, 2018) would shed more light on this issue.

Human studies suggest that cognitive factors may also explain immature behavior. Individual children often show substantial fluctuations in sensory thresholds within a single training session and erratic learning trajectories (Halliday et al., 2008). Similar findings have been observed in animal models (Green et al., 2016) and are taken as evidence of pronounced fluctuations of the internal state. In addition, infants and children often display poorer asymptotic performance than adults on psychometric auditory detection and discrimination tasks, suggesting an inability to maintain their focus (Bargones et al., 1995; Halliday et al., 2008). Given that attention plays an important role in perceptual learn- 
ing (Shiu and Pashler, 1992; Ahissar and Hochstein, 1993; Polley et al., 2006; Mukai et al., 2011; Szpiro and Carrasco, 2015), it is conceivable that immature attentional resources or strategies contribute to more variable sensory representations and learning trajectories during development.

The maturational state of nonsensory mechanisms could plausibly explain why adolescent neurons respond differently to perceptual training. Processes such as attention (McAdams and Maunsell, 1999; Petkov et al., 2004; Herrero et al., 2008; Cohen and Maunsell, 2010), arousal (McGinley et al., 2015a,b), reward (David et al., 2012), and expectation (Kok et al., 2012; Buran et al., 2014b; Gavornik and Bear, 2014; Carcea et al., 2017) have all been shown to have a direct impact on sensory gain, and are thus likely mechanisms by which perceptual training enhances signalto-noise ratios. Indeed, our previous findings suggest that perceptual training strengthens the top-down networks that modulate auditory cortical response properties (Caras and Sanes, 2017), and a similar mechanism may support visual perceptual learning (Schäfer et al., 2007; Bartolucci and Smith, 2011; Byers and Serences, 2012, 2014). Nonsensory mechanisms also influence trial-to-trial variability of sensory-evoked cortical responses (Mitchell et al., 2007, 2009; Cohen and Maunsell, 2009; Goris et al., 2014; McGinley et al., 2015a; von Trapp et al., 2016). For example, attended stimuli elicit more reliable responses from macaque V4 neurons (Mitchell et al., 2007). Similarly, an optimal arousal level is significantly correlated with a lower variance of auditory cortical neuron membrane potential (McGinley et al., 2015a).

The anatomy (Rakic et al., 1986; Huttenlocher and Dabholkar, 1997; Giedd et al., 1999; Sowell et al., 2002), activation (Galvan et al., 2006; Qu et al., 2015), and functional connectivity (Gee et al., 2013; Fareri et al., 2015; Heller et al., 2016) of the neural pathways that subserve nonsensory mechanisms undergo a protracted development, with pronounced changes during adolescence (for review, see Somerville et al., 2010; Selemon, 2013; Larsen and Luna, 2018). For example, longitudinal imaging has shown that the volume of frontal lobe gray matter peaks at $\sim 12$ years of age in humans (Giedd et al., 1999). Similarly, Galvan et al., 2006 found that adolescents display greater reward-based activity in the nucleus accumbens compared with children or adults. Thus, immature or unstable top-down functionality may contribute to the diminished capacity for signal-to-noise improvements, and immature neuromodulatory circuits may contribute to larger response variance in adolescent animals.

\section{Relationship to previous developmental findings in gerbils}

Perceptual learning of developing gerbils has been examined previously using an AM depth detection paradigm that is nearly identical to the one we described here (Sarro and Sanes, 2010). Consistent with our current findings, Sarro and Sanes (2010) found that adolescent animals improved more slowly than adults. However, there were several methodological differences between their study and ours. First, the criterion for procedural training was somewhat more stringent for the present study: animals were required to complete at least three procedural training sessions, achieving a $d^{\prime}$ of $\geq 1.5$ in at least one session, while Sarro and Sanes (2010) only required animals to obtain 70\% correct over 10 consecutive trials. Therefore, it is possible that animals in the current study were better trained on the task before perceptual training began. Second, the AM stimuli were presented in a different manner in each study. For example, Sarro and Sanes (2010) used a randomized stimulus presentation order, with a larger range of AM depths, to assess naive thresholds. This ap- proach induces greater uncertainty about the stimulus compared with the method we used here (a limited number of depths spanning threshold, presented in a descending order). In addition, Sarro and Sanes (2010) presented warn trials more rapidly than we did (separating them by two to four safe trials, rather than three to five safe stimuli, as we did here). Both of these factors may have resulted in a more attentionally demanding task. Given that attentional capabilities are slow to mature, as discussed above, these methodological differences may have led to the relatively poor learning that Sarro and Sanes (2010) observed in all immature animals. A final key difference worth highlighting is the amount of practice each subject received on the task. Subjects in the Sarro and Sanes (2010) study performed fewer trials per session (20-50 vs our 67-81), and fewer sessions overall ( $5 \mathrm{~d}$ vs our $10 \mathrm{~d}$ ). Thus, young (i.e., preadolescent) subjects may require more than a minimum amount of training to obtain adult-like levels of performance and improvement.

\section{Conclusion}

Collectively, our findings support the idea that the development of perceptual learning on an AM detection task is nonmonotonic, displaying a temporary impairment during the adolescent period. This adolescent behavioral phenotype is associated with an increased similarity between signal-evoked and noise-evoked neural responses, and greater trial-by-trial response fluctuations, both of which limit training-based improvements in auditory cortical sensitivity. Together, these findings suggest that heightened neural variability during adolescence limits perceptual learning and raise the possibility that other forms of implicit skill learning are subject to similar constraints.

\section{References}

Adab HZ, Vogels R (2011) Practicing coarse orientation discrimination improves orientation signals in macaque cortical area v4. Curr Biol 21:16611666.

Adolph KE, Hoch JE, Cole WG (2018) Development (of walking): 15 suggestions. Trends Cogn Sci 22:699-711.

Ahissar M, Hochstein S (1993) Attentional control of early perceptual learning. Proc Natl Acad Sci U S A 90:5718-5722.

Allen P, Wightman F (1994) Psychometric functions for children's detection of tones in noise. J Speech Hear Res 37:205-215.

Amitay S, Zhang YX, Jones PR, Moore DR (2014) Perceptual learning: top to bottom. Vision Res 99:69-77.

Bao S, Chang EF, Woods J, Merzenich MM (2004) Temporal plasticity in the primary auditory cortex induced by operant perceptual learning. Nat Neurosci 7:974-981.

Baptista LF, Morton ML (1981) Interspecific song acquisition by a whitecrowned sparrow. Auk 98:383-385.

Bargones JY, Werner LA, Marean GC (1995) Infant psychometric functions for detection: mechanisms of immature sensitivity. J Acoust Soc Am 98: 99-111.

Bartolucci M, Smith AT (2011) Attentional modulation in visual cortex is modified during perceptual learning. Neuropsychologia 49:3898-3907.

Beitel RE, Schreiner CE, Cheung SW, Wang X, Merzenich MM (2003) Reward-dependent plasticity in the primary auditory cortex of adult monkeys trained to discriminate temporally modulated signals. Proc Natl Acad Sci U S A 100:11070-11075.

Brown TT (2017) Individual differences in human brain development. Wiley Interdiscip Rev Cogn Sci 8:e1389.

Buran BN, Sarro EC, Manno FA, Kang R, Caras ML, Sanes DH (2014a) A sensitive period for the impact of hearing loss on auditory perception. J Neurosci 34:2276-2284.

Buran BN, von Trapp G, Sanes DH (2014b) Behaviorally gated reduction of spontaneous discharge can improve detection thresholds in auditory cortex. J Neurosci 34:4076-4081.

Buss E, Hall JW 3rd, Grose JH (2006) Development and the role of internal noise in detection and discrimination thresholds with narrow band stimuli. J Acoust Soc Am 120:2777-2788. 
Buss E, Hall JW 3rd, Grose JH (2009) Psychometric functions for pure tone intensity discrimination: slope differences in school-aged children and adults. J Acoust Soc Am 125:1050-1058.

Byers A, Serences JT (2012) Exploring the relationship between perceptual learning and top-down attentional control. Vision Res 74:30-39.

Byers A, Serences JT (2014) Enhanced attentional gain as a mechanism for generalized perceptual learning in human visual cortex. J Neurophysiol 112:1217-1227.

Camp LL, Rudy JW (1988) Changes in the categorization of appetitive and aversive events during postnatal development of the rat. Dev Psychobiol 21:25-42.

Caras ML (2013) Estrogenic modulation of auditory processing: a vertebrate comparison. Front Neuroendocrinol 34:285-299.

Caras ML, Sanes DH (2015) Sustained perceptual deficits from transient sensory deprivation. J Neurosci 35:10831-10842.

Caras ML, Sanes DH (2017) Top-down modulation of sensory cortex gates perceptual learning. Proc Natl Acad Sci U S A 114:9972-9977.

Carcea I, Insanally MN, Froemke RC (2017) Dynamics of auditory cortical activity during behavioural engagement and auditory perception. Nat Commun 8:14412.

Carey S, Diamond R, Woods B (1980) Development of face recognition-a maturational component? Dev Psychol 16:257-269.

Cauffman E, Shulman EP, Steinberg L, Claus E, Banich MT, Graham S, Woolard J (2010) Age differences in affective decision making as indexed by performance on the Iowa gambling task. Dev Psychol 46:193207.

Chihak BJ, Grechkin TY, Kearney JK, Cremer JF, Plumert JM (2014) How children and adults learn to intercept moving gaps. J Exp Child Psychol 122:134-152.

Clark MM, Galef BG Jr (1980) Effects of rearing environment on adrenal weights, sexual development, and behavior in gerbils: an examination of richter's domestication hypothesis. J Comp Physiol Psychol 94:857-863.

Clayton NS (1987) Song learning in bengalese finches: a comparison with zebra finches. Ethology 76:247-255.

Cohen MR, Maunsell JH (2009) Attention improves performance primarily by reducing interneuronal correlations. Nat Neurosci 12:1594-1600.

Cohen MR, Maunsell JH (2010) A neuronal population measure of attention predicts behavioral performance on individual trials. J Neurosci 30: 15241-15253.

Crist RE, Li W, Gilbert CD (2001) Learning to see: experience and attention in primary visual cortex. Nat Neurosci 4:519-525.

David SV, Fritz JB, Shamma SA (2012) Task reward structure shapes rapid receptive field plasticity in auditory cortex. Proc Natl Acad Sci U S A 109:2144-2149.

Dean KF, Sheets LP, Crofton KM, Reiter LW (1990) The effect of age and experience on inhibition of the acoustic startle response by gaps in background noise. Psychobiology 18:89-95.

Decker JH, Lourenco FS, Doll BB, Hartley CA (2015) Experiential reward learning outweighs instruction prior to adulthood. Cogn Affect Behav Neurosci 15:310-320.

Decker JH, Otto AR, Daw ND, Hartley CA (2016) From creatures of habit to goal-directed learners: tracking the developmental emergence of modelbased reinforcement learning. Psychol Sci 27:848-858.

Dosher BA, Lu ZL (1998) Perceptual learning reflects external noise filtering and internal noise reduction through channel reweighting. Proc Natl Acad Sci U S A 95:13988-13993.

Dosher BA, Lu ZL (1999) Mechanisms of perceptual learning. Vision Res 39:3197-3221.

Fareri DS, Gabard-Durnam L, Goff B, Flannery J, Gee DG, Lumian DS, Caldera C, Tottenham N (2015) Normative development of ventral striatal resting state connectivity in humans. Neuroimage 118:422-437.

Friedman JT, Peiffer AM, Clark MG, Benasich AA, Fitch RH (2004) Age and experience-related improvements in gap detection in the rat. Brain Res Dev Brain Res 152:83-91.

Galvan A, Hare TA, Parra CE, Penn J, Voss H, Glover G, Casey BJ (2006) Earlier development of the accumbens relative to orbitofrontal cortex might underlie risk-taking behavior in adolescents. J Neurosci 26:68856892.

Gavornik JP, Bear MF (2014) Learned spatiotemporal sequence recognition and prediction in primary visual cortex. Nat Neurosci 17:732-737.

Gee DG, Humphreys KL, Flannery J, Goff B, Telzer EH, Shapiro M, Hare TA, Bookheimer SY, Tottenham N (2013) A developmental shift from pos- itive to negative connectivity in human amygdala-prefrontal circuitry. J Neurosci 33:4584-4593.

Gibson EJ (1953) Improvement in perceptual judgments as a function of controlled practice or training. Psychol Bull 50:401-431.

Giedd JN, Blumenthal J, Jeffries NO, Castellanos FX, Liu H, Zijdenbos A, Paus T, Evans AC, Rapoport JL (1999) Brain development during childhood and adolescence: a longitudinal MRI study. Nat Neurosci 2:861-863.

Goris RL, Movshon JA, Simoncelli EP (2014) Partitioning neuronal variability. Nat Neurosci 17:858-865.

Green DB, Ohlemacher J, Rosen MJ (2016) Benefits of stimulus exposure: developmental learning independent of task performance. Front Neurosci 10:263.

Green DM, Swets JA (1966) Signal detection theory and psychophysics. New York: Wiley.

Gu Y, Liu S, Fetsch CR, Yang Y, Fok S, Sunkara A, DeAngelis GC, Angelaki DE (2011) Perceptual learning reduces interneuronal correlations in macaque visual cortex. Neuron 71:750-761

Halliday LF, Taylor JL, Edmondson-Jones AM, Moore DR (2008) Frequency discrimination learning in children. J Acoust Soc Am 123:4393-4402.

Hartley CA, Somerville LH (2015) The neuroscience of adolescent decisionmaking. Curr Opin Behav Sci 5:108-115.

Hartley DE, Wright BA, Hogan SC, Moore DR (2000) Age-related improvements in auditory backward and simultaneous masking in 6- to 10-yearold children. J Speech Lang Hear Res 43:1402-1415.

Heffner HE, Heffner RS (1984) Temporal lobe lesions and perception of species-specific vocalizations by macaques. Science 226:75-76.

Heller AS, Cohen AO, Dreyfuss MF, Casey BJ (2016) Changes in corticosubcortical and subcortico-subcortical connectivity impact cognitive control to emotional cues across development. Soc Cogn Affect Neurosci 11:1910-1918.

Herrero JL, Roberts MJ, Delicato LS, Gieselmann MA, Dayan P, Thiele A (2008) Acetylcholine contributes through muscarinic receptors to attentional modulation in v1. Nature 454:1110-1114

Hill DN, Mehta SB, Kleinfeld D (2011) Quality metrics to accompany spike sorting of extracellular signals. J Neurosci 31:8699-8705.

Huttenlocher PR, Dabholkar AS (1997) Regional differences in synaptogenesis in human cerebral cortex. J Comp Neurol 387:167-178.

Huyck JJ, Wright BA (2011) Late maturation of auditory perceptual learning. Dev Sci 14:614-621.

Huyck JJ, Wright BA (2013) Learning, worsening, and generalization in response to auditory perceptual training during adolescence. J Acoust Soc Am 134:1172-1182.

Irvine DRF (2018) Auditory perceptual learning and changes in the conceptualization of auditory cortex. Hear Res 366:3-16.

Jamison HL, Watkins KE, Bishop DV, Matthews PM (2006) Hemispheric specialization for processing auditory nonspeech stimuli. Cereb Cortex 16:1266-1275.

Jones PR, Moore DR, Amitay S, Shub DE (2013) Reduction of internal noise in auditory perceptual learning. J Acoust Soc Am 133:970-981.

Kahnt T, Grueschow M, Speck O, Haynes JD (2011) Perceptual learning and decision-making in human medial frontal cortex. Neuron 70:549559.

King EC, Pattwell SS, Sun A, Glatt CE, Lee FS (2013) Nonlinear developmental trajectory of fear learning and memory. Ann N Y Acad Sci 1304: 62-69.

King EC, Pattwell SS, Glatt CE, Lee FS (2014) Sensitive periods in fear learning and memory. Stress 17:13-21.

Knoll LJ, Fuhrmann D, Sakhardande AL, Stamp F, Speekenbrink M, Blakemore SJ (2016) A window of opportunity for cognitive training in adolescence. Psychol Sci 27:1620-1631.

Kok P, Jehee JF, de Lange FP (2012) Less is more: expectation sharpens representations in the primary visual cortex. Neuron 75:265-270.

Kuhl PK (2000) A new view of language acquisition. Proc Natl Acad Sci U S A 97:11850-11857.

Kuhl PK, Stevens E, Hayashi A, Deguchi T, Kiritani S, Iverson P (2006) Infants show a facilitation effect for native language phonetic perception between 6 and 12 months. Dev Sci 9:F13-F21.

Larsen B, Luna B (2018) Adolescence as a neurobiological critical period for the development of higher-order cognition. Neurosci Biobehav Rev 94: 179-195. 
Law CT, Gold JI (2008) Neural correlates of perceptual learning in a sensory-motor, but not a sensory, cortical area. Nat Neurosci 11:505-513.

Li W, Piëch V, Gilbert CD (2008) Learning to link visual contours. Neuron $57: 442-451$

Lloyd RS, Oliver JL, Faigenbaum AD, Myer GD, De Ste Croix MB (2014) Chronological age vs. biological maturation: implications for exercise programming in youth. J Strength Cond Res 28:1454-1464.

Lu ZL, Hua T, Huang CB, Zhou Y, Dosher BA (2011) Visual perceptual learning. Neurobiol Learn Mem 95:145-151.

Ludwig KA, Miriani RM, Langhals NB, Joseph MD, Anderson DJ, Kipke DR (2009) Using a common average reference to improve cortical neuron recordings from microelectrode arrays. J Neurophysiol 101:1679-1689.

Lukács Á, Kemény F (2015) Development of different forms of skill learning throughout the lifespan. Cogn Sci 39:383-404.

Lyamzin D, Benucci A (2018) The mouse posterior parietal cortex: anatomy and functions. Neurosci Res. Advance online publication. Retrieved February 6, 2018. doi:10.1016/j.neures.2018.10.008.

Macmillan NA, Creelman CD (1990) Response bias: characteristics of detection theory, threshold theory, and "nonparametric" indexes. Psychol Bull 107:401.

Marler P, Tamura M (1964) Culturally transmitted patterns of vocal behavior in sparrows. Science 146:1483-1486.

Marshall WA, Tanner JM (1969) Variations in pattern of pubertal changes in girls. Arch Dis Child 44:291-303.

McAdams CJ, Maunsell JH (1999) Effects of attention on the reliability of individual neurons in monkey visual cortex. Neuron 23:765-773.

McCallum J, Kim JH, Richardson R (2010) Impaired extinction retention in adolescent rats: effects of d-cycloserine. Neuropsychopharmacology 35 : $2134-2142$.

McGinley MJ, David SV, McCormick DA (2015a) Cortical membrane potential signature of optimal states for sensory signal detection. Neuron $87: 179-192$

McGinley MJ, Vinck M, Reimer J, Batista-Brito R, Zagha E, Cadwell CR, Tolias AS, Cardin JA, McCormick DA (2015b) Waking state: rapid variations modulate neural and behavioral responses. Neuron 87:1143-1161.

Mitchell JF, Sundberg KA, Reynolds JH (2007) Differential attentiondependent response modulation across cell classes in macaque visual area v4. Neuron 55:131-141.

Mitchell JF, Sundberg KA, Reynolds JH (2009) Spatial attention decorrelates intrinsic activity fluctuations in macaque area v4. Neuron 63:879888 .

Mogil JS (1999) The genetic mediation of individual differences in sensitivity to pain and its inhibition. Proc Natl Acad Sci U S A 96:7744-7751.

Moore DR, Ferguson MA, Halliday LF, Riley A (2008) Frequency discrimination in children: perception, learning and attention. Hear Res 238:147154.

Moore DR, Cowan JA, Riley A, Edmondson-Jones AM, Ferguson MA (2011) Development of auditory processing in 6- to 11-yr-old children. Ear Hear 32:269-285

Moye TB, Rudy JW (1987) Otogenesis of trace conditioning in young rats: dissociation of associative and memory processes. Dev Psychobiol 20: 405-414.

Mukai I, Bahadur K, Kesavabhotla K, Ungerleider LG (2011) Exogenous and endogenous attention during perceptual learning differentially affect post-training target thresholds. J Vis 11:25, 1-15.

Ortiz JA, Wright BA (2009) Contributions of procedure and stimulus learning to early, rapid perceptual improvements. J Exp Psychol Hum Percept Perform 35:188-194.

Overman WH (1990) Performance on traditional matching to sample, non-matching to sample, and object discrimination tasks by 12- to 32month-old children. A developmental progression. Ann N Y Acad Sci 608:365-385.

Pattwell SS, Bath KG, Casey BJ, Ninan I, Lee FS (2011) Selective earlyacquired fear memories undergo temporary suppression during adolescence. Proc Natl Acad Sci U S A 108:1182-1187.

Pattwell SS, Duhoux S, Hartley CA, Johnson DC, Jing D, Elliott MD, Ruberry EJ, Powers A, Mehta N, Yang RR, Soliman F, Glatt CE, Casey BJ, Ninan I, Lee FS (2012) Altered fear learning across development in both mouse and human. Proc Natl Acad Sci U S A 109:16318-16323.

Pattwell SS, Lee FS, Casey BJ (2013) Fear learning and memory across adolescent development: hormones and behavior special issue: puberty and adolescence. Horm Behav 64:380-389.
Petkov CI, Kang X, Alho K, Bertrand O, Yund EW, Woods DL (2004) Attentional modulation of human auditory cortex. Nat Neurosci 7:658-663.

Petrov AA, Dosher BA, Lu ZL (2005) The dynamics of perceptual learning: an incremental reweighting model. Psychol Rev 112:715-743.

Pinto-Fochi ME, Negrin AC, Scarano WR, Taboga SR, Góes RM (2016) Sexual maturation of the mongolian gerbil (meriones unguiculatus): a histological, hormonal and spermatic evaluation. Reprod Fertil Dev 28:815-823.

Polley DB, Steinberg EE, Merzenich MM (2006) Perceptual learning directs auditory cortical map reorganization through top-down influences. J Neurosci 26:4970-4982.

Powers AR 3rd, Hevey MA, Wallace MT (2012) Neural correlates of multisensory perceptual learning. J Neurosci 32:6263-6274.

Qu Y, Galvan A, Fuligni AJ, Lieberman MD, Telzer EH (2015) Longitudinal changes in prefrontal cortex activation underlie declines in adolescent risk taking. J Neurosci 35:11308-11314.

Quiroga RQ, Nadasdy Z, Ben-Shaul Y (2004) Unsupervised spike detection and sorting with wavelets and superparamagnetic clustering. Neural Comput 16:1661-1687.

Radtke-Schuller S, Schuller G, Angenstein F, Grosser OS, Goldschmidt J, Budinger E (2016) Brain atlas of the Mongolian gerbil (meriones unguiculatus) in CT/MRI-aided stereotaxic coordinates. Brain Struct Funct 221 [Suppl 1]:1-272.

Raiguel S, Vogels R, Mysore SG, Orban GA (2006) Learning to see the difference specifically alters the most informative v4 neurons. J Neurosci 26:6589-6602.

Rakic P, Bourgeois JP, Eckenhoff MF, Zecevic N, Goldman-Rakic PS (1986) Concurrent overproduction of synapses in diverse regions of the primate cerebral cortex. Science 232:232-235.

Recanzone GH, Merzenich MM, Jenkins WM, Grajski KA, Dinse HR (1992) Topographic reorganization of the hand representation in cortical area $3 \mathrm{~b}$ owl monkeys trained in a frequency-discrimination task. J Neurophysiol 67:1031-1056.

Recanzone GH, Schreiner CE, Merzenich MM (1993) Plasticity in the frequency representation of primary auditory cortex following discrimination training in adult owl monkeys. J Neurosci 13:87-103.

Sanders JO, Qiu X, Lu X, Duren DL, Liu RW, Dang D, Menendez ME, Hans SD, Weber DR, Cooperman DR (2017) The uniform pattern of growth and skeletal maturation during the human adolescent growth spurt. Sci Rep 7:16705.

Sarro EC, Sanes DH (2010) Prolonged maturation of auditory perception and learning in gerbils. Dev Neurobiol 70:636-648.

Sarro EC, Sanes DH (2011) The cost and benefit of juvenile training on adult perceptual skill. J Neurosci 31:5383-5391.

Schäfer R, Vasilaki E, Senn W (2007) Perceptual learning via modification of cortical top-down signals. PLoS Comput Biol 3:e165.

Schoups A, Vogels R, Qian N, Orban G (2001) Practising orientation identification improves orientation coding in v1 neurons. Nature 412:549-553.

Schütt HH, Harmeling S, Macke JH, Wichmann FA (2016) Painfree and accurate bayesian estimation of psychometric functions for (potentially) overdispersed data. Vision Res 122:105-123.

Selemon LD (2013) A role for synaptic plasticity in the adolescent development of executive function. Transl Psychiatry 3:e238.

Shiu LP, Pashler H (1992) Improvement in line orientation discrimination is retinally local but dependent on cognitive set. Percept Psychophys 52: 582-588.

Siegford JM, Hadi Mansouri S, Ulibarri C (2003) Normal ontogeny of perineal muscles and testosterone levels in mongolian gerbils; response to testosterone in developing females. Anat Rec A Discov Mol Cell Evol Biol 275:997-1008.

Sisneros JA (2009) Steroid-dependent auditory plasticity for the enhancement of acoustic communication: recent insights from a vocal teleost fish. Hear Res 252:9-14.

Somerville LH, Jones RM, Casey BJ (2010) A time of change: behavioral and neural correlates of adolescent sensitivity to appetitive and aversive environmental cues. Brain Cogn 72:124-133.

Somerville LH, Hare T, Casey BJ (2011) Frontostriatal maturation predicts cognitive control failure to appetitive cues in adolescents. J Cogn Neurosci 23:2123-2134.

Sowell ER, Trauner DA, Gamst A, Jernigan TL (2002) Development of cortical and subcortical brain structures in childhood and adolescence: a structural MRI study. Dev Med Child Neurol 44:4-16. 
Szpiro SF, Carrasco M (2015) Exogenous attention enables perceptual learning. Psychol Sci 26:1854-1862.

van der Schaaf ME, Warmerdam E, Crone EA, Cools R (2011) Distinct linear and non-linear trajectories of reward and punishment reversal learning during development: relevance for dopamine's role in adolescent decision making. Dev Cogn Neurosci 1:578-590.

von Trapp G, Buran BN, Sen K, Semple MN, Sanes DH (2016) A decline in response variability improves neural signal detection during auditory task performance. J Neurosci 36:11097-11106.

Wakefield GH, Viemeister NF (1990) Discrimination of modulation depth of sinusoidal amplitude modulation (sam) noise. J Acoust Soc Am 88:1367-1373.

Walker KM, Hall SE, Klein RM, Phillips DP (2006) Development of perceptual correlates of reading performance. Brain Res 1124:126-141.
Wetzel W, Ohl FW, Scheich H (2008) Global versus local processing of frequency-modulated tones in gerbils: an animal model of lateralized auditory cortex functions. Proc Natl Acad Sci U S A 105:6753-6758.

Woolley SM, Hauber ME, Theunissen FE (2010) Developmental experience alters information coding in auditory midbrain and forebrain neurons. Dev Neurobiol 70:235-252.

Wright BA, Zhang Y (2009) Insights into human auditory processing gained from perceptual learning. In: The cognitive neurosciences (Gazzaniga MS, ed), pp 353-366. Cambridge, MA: MIT Press.

Yan Y, Rasch MJ, Chen M, Xiang X, Huang M, Wu S, Li W (2014) Perceptual training continuously refines neuronal population codes in primary visual cortex. Nat Neurosci 17:1380-1387.

Yang T, Maunsell JH (2004) The effect of perceptual learning on neuronal responses in monkey visual area v4. J Neurosci 24:1617-1626. 